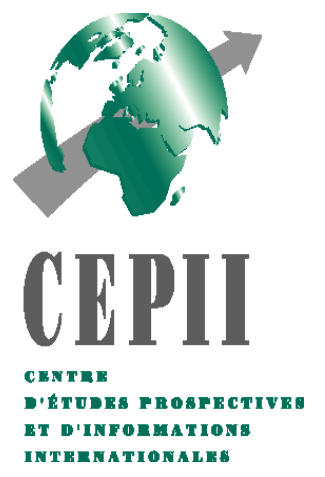

No $1994-12$
Novembre

\title{
Croissance et spécialisation
}

Frédéric Busson

Pierre Villa 


\section{SOMMAIRE}

Résumé

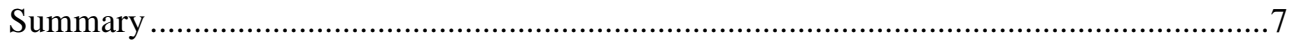

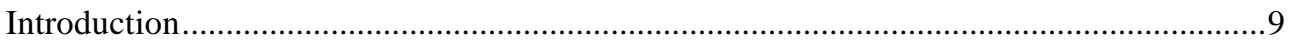

1.Trois indices du commerce extérieur....................................................................... 10

1.1. L'indice de spécialisation interbranche du commerce......................................10

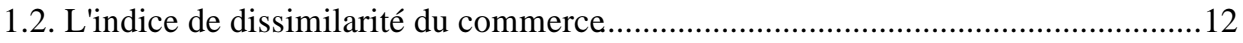

1.3. L'indice de croissance de la demande étrangère...................................................13

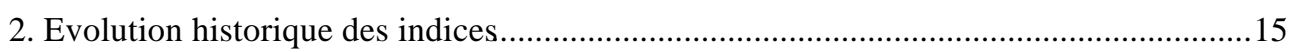

3. Les résultats économétriques.....................................................................................18

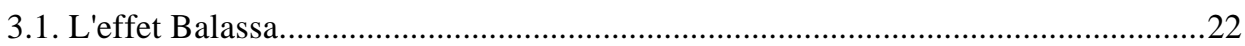

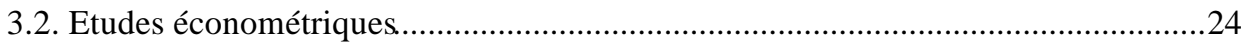

3.2.1. Régressions avec l'indice de spécialisation interbranche.........................26

3.2.2. Régressions avec l'indice de dissimilarité du commerce...........................27

3.2.3. Régressions avec l'indice de croissance de la demande étrangère.............29

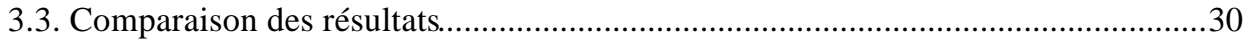

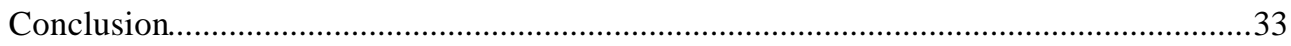

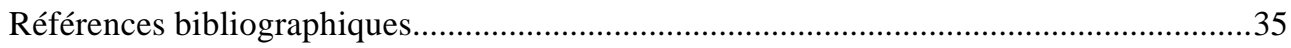

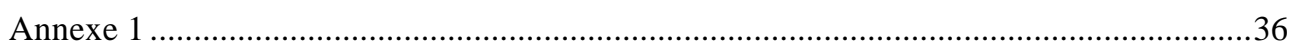

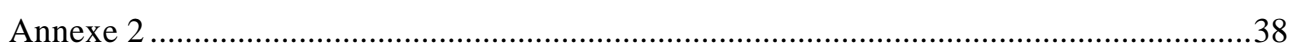

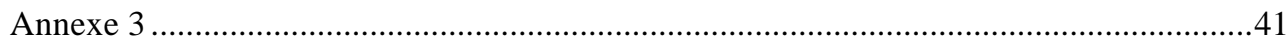

Liste des documents de travail du CEPII......................................................................44 



\section{Résumé}

L'intensification du commerce Nord-Sud, les délocalisations d'activités, l'accroissement de la mobilité du capital, de même que l'abandon des stratégies de substitution à l'importation et l'ouverture sur l'extérieur des anciens pays à économie planifiée nous amènent à nous interroger sur les liens entre commerce extérieur et croissance économique. Pour cela, nous introduisons des variables de commerce international dans le modèle de convergence de Barro et Sala-I-Martin (1991), ainsi que la diversité des biens capitaux dans l'esprit de Ethier (1982) et Romer (1987). Cela nous amène en particulier à construire et discuter trois indices: l'indice de spécialisation interbranche du commerce, l'indice de dissimilarité prenant en compte l'incertitude de la croissance des exportations en volume et l'indice de croissance de la demande étrangère mesurant les interactions de la spécialisation interbranche et la croissance du marché mondial.

L'ouverture d'un pays au commerce international permet d'exploiter des avantages comparatifs et de développer des secteurs où il y a des économies d'échelle pour acquérir, en contrepartie, des technologies étrangères plus productives et des biens de consommation meilleur marché. Ainsi, selon ses dotations factorielles, sa richesse initiale et sa politique économique, un pays choisira, à l'instar du Japon, une forte spécialisation interbranche ou, à l'inverse, développera, à l'image de la plupart des pays européens, un commerce intrabranche sans spécialisation sectorielle marquée. Au delà de l'ouverture même, la nature de la spécialisation du commerce apparaît donc comme un facteur susceptible d'influencer la croissance.

D'autres facteurs méritent aussi attention. La sous-évaluation du taux de change devrait favoriser le développement d'un secteur exportateur efficace par effet compétitivité ou profitabilité au détriment du secteur abrité moins à même d'augmenter sa productivité. La volatilité des termes de l'échange devrait, quant à elle, rendre plus aléatoire les rendements à l'exportation et entraver la production nationale en entachant d'incertitude les coûts des équipements et des matières premières importés. Enfin, la demande internationale, lorsqu'elle est vigoureuse dans les secteurs où le pays à réussi à se positionner comme exportateur net, devrait stimuler sa croissance.

Les résultats auxquels nous parvenons sont les suivants

- La sous-évaluation du taux de change stimule très légèrement la croissance.

- La volatilité des termes de l'échange n'a pas d'influence.

- L'ouverture d'une économie aux échanges internationaux est de nature à favoriser la croissance dans les deux cas suivants:

- soit le pays réussit à se positionner sur quelques secteurs où la demande mondiale est forte et parvient à suivre leur évolution; il réalise alors une bonne spécialisation interbranche qui tire sa croissance ; s'en suivra alors, avec son développement économique, une augmentation naturelle de son commerce intrabranche qui évincera progressivement sa forte spécialisation interbranche initiale ; toutefois, peu de pays ont réussi dans cette voie : citons le cas du Japon et des Dragons; 


\section{Croissance et spécialisation}

- soit le pays ne parvient pas à se spécialiser dans de tels secteurs et alors il gagnera à développer un commerce fortement intrabranche de biens capitaux, ce qui lui apportera une grande diversité dans ces biens ainsi qu'une forte productivité globale, deux effets très bénéfiques sur sa croissance économique; il évitera alors le piège d'une spécialisation interbranche excessive dans lequel sont tombés beaucoup de pays d'Afrique ou d'Amérique latine. 


\section{Summary}

The intensification of North-South trade, the re-location of production, and the rising mobility of capital, along with the abandonment of import substitution strategies and the opening up of the formerly, centrally planned economies have led us to examine the links between foreign trade and economic growth. For this purpose, we have introduced variables describing the pattern of international trade into Barro and Sala-I-Martin's (1991) convergence model, as well as the diversity of capital goods using the approach of Ethier (1982) and Romer (1987). This has led us to construct and discuss three indices: the intersectoral trade index, the dissimilarity index which takes account of the uncertainty in the real growth of exports, and the foreign demand growth index which measures the interaction between inter-sectoral specialisation and the growth of world trade.

The opening up of a country to international trade allows it to exploit its comparative advantages, and develop sectors in which economies of scale may be achieved, in exchange for more productive foreign technology and cheaper consumer goods. Given its natural endowments, initial wealth and economic policy, a country may choose to develop a strong inter-sectoral specialisation, as was the case of Japan, or follow the course of the European countries which are characterised by marked intra-sectoral specialisation. The process of trade specialisation itself thus appears to be a factor likely to influence growth.

Other factors also merit attention. Exchange rate under-valuation should favour the development of an efficient export sector due to competitiveness or profitability effects, while at the same time penalising the non-tradable sector which is less able to achieve large productivity gains. Terms of trade volatility, on the other hand, should render the benefits of trade less certain, and may slow down domestic growth because of uncertainties over the prices of imported capital equipment and raw materials. Lastly, strong international demand, in those areas in which a country has positioned itself as a net exporter, ought clearly to stimulate growth.

The results of our research are that:

- exchange rate under-valuation stimulates growth slightly;

- terms of trade volatility has no impact on growth;

- the opening up of an economy to international trade will favour growth in the following two cases:

-either the country succeeds in positioning itself in certain sectors for which world demand is strong, and manages to keep up with the evolution of these sectors. In this case, successful inter-sectoral specialisation pulls along growth. As the country develops, its intra-sectoral trade will rise, and this will ultimately outweigh its strong inter-sectoral specialisation. However, few countries have successfully followed this path, notably Japan and the Asian Dragons;

-or a country does not manage to specialise in particular sectors but will benefit from strongly developing intra-sectoral trade in capital equipment goods. This will lead to a great diversity in such goods, as well as high levels of productivity output, both of which are 


\section{Croissance et spécialisation}

beneficial for the economy. Thus a country will be able to avoid the trap of excessive intersectoral specialisation, which has plagued a large number of African and Latin American countries. 


\title{
CROISSSANCE ET SPECIALISATION
}

\author{
Frédéric Busson ${ }^{1}$ et Pierre Villa 2
}

\section{Introduction}

La croissance différentielle des économies est souvent associée à leur degré d'ouverture. Selon les arguments traditionnels, le fait d'orienter l'économie vers l'extérieur permet de profiter des avantages comparatifs et de développer les secteurs où il y a des économies d'échelle à condition de se spécialiser dans les domaines où la croissance de la demande mondiale est la plus forte. D'autres arguments plus macro-économiques ou plus spécifiques aux pays en développement sont aussi évoqués. Un taux de change réel bas permet d'accroître les exportations par effet compétitivité. Le développement des exportations desserre la contrainte extérieure et permet d'importer du capital non produit nationalement, ce qui favorise la croissance. A l'inverse, un taux de change réel élevé favorise le secteur traditionnel (pour les PED) ou le secteur abrité (pour les pays développés). En revanche une grande variabilité du taux de change accroît l'incertitude sur les rendements à l'exportation et risque d'entraver ces dernières en faisant jouer un rôle important à la profitabilité à l'exportation lorsque les producteurs nationaux ont de l'aversion pour le risque. Enfin une argumentation plus stratégique invoque la spécialisation comme un des facteurs de la croissance. Les pays devraient se spécialiser dans les secteurs où ils ont un avantage comparatif lié à leur dotation factorielle (spécialisation de type Heckscher-Ohlin) ce qui renvoie à une spécialisation interbranche, ou se spécialiser dans des produits plus spécifiques pour se créer des niches et profiter des rendements d'échelle (spécialisation de type intrabranche). Les faits ne donnent pas de réponses claires : parmi les pays développés, le Japon présente une spécialisation interbranche "forte", les pays européens une spécialisation "faible" ; parmi les pays en développement, l'Afrique est très spécialisée (autant que le Japon) alors que les Nouveaux Pays Industrialisés (Dragons) ont une spécialisation interbranche intermédiaire. La nature de la spécialisation importe donc.

L'objet de cet article est d'étudier les liens entre commerce extérieur et croissance. Après avoir introduit les facteurs habituels (voir Barro (1991) et Mankiw, Romer et Weil (1991)) : richesse initiale pour la convergence, capital physique, capital humain, nous introduisons des variables explicatives liées au commerce international. Cela nous permet de mettre en évidence les faits suivants:

- la sous-évaluation du taux de change a une influence qui dépend toutefois de la géographie et du degré de convertibilité des monnaies,

- la spécialisation interbranche a en général un effet négatif sur la croissance sauf lorsqu'elle a été orientée vers les quelques secteurs qui ont connu un développement rapide

- l'adéquation de la spécialisation à la demande mondiale favorise la croissance

- la volatilité des termes de l'échange n'a pas d'influence

- la croissance est orientée à l'exportation, les stratégies de substitution à l'importation ayant joué un rôle secondaire.

\footnotetext{
${ }^{1}$ Economiste au CEPII.

${ }^{2}$ Conseiller scientifique au CEPII.
} 
Ces résultats incitent à penser que la spécialisation interbranche est plus souvent une donnée de fait pour les pays qu'une construction et que les politiques de changes flexibles associées à une instabilité monétaire ont peu d'influence.

Le texte est organisé en trois parties : tout d'abord nous expliquons comment sont construits les indices du commerce extérieur, ensuite nous procédons à une analyse descriptive, puis nous commentons les résultats économétriques.

\section{Trois indices du commerce extérieur}

\subsection{L'indice de spécialisation interbranche du commercể}

Le but est de construire un indice de spécialisation du commerce extérieur d'un pays qui soit indépendant des effets conjoncturels et du solde commercial. L'idée intuitive de départ est qu'un pays est fortement spécialisé si son commerce extérieur peut être partitionné en branches où le pays est soit fortement exportateur, soit fortement importateur alors que son commerce extérieur est globalement équilibré. Toutefois, dans la réalité, le commerce extérieur n'est pas équilibré. Or certaines théories attribuent un excédent commercial à une "bonne" spécialisation en termes de facteurs de production (spécialisation de type Heckscher-Ohlin) ou en termes de position du pays dans les secteurs où la demande mondiale croît le plus rapidement. D'autres l'attribuent à des phénomènes conjoncturels ou macro-économiques, que le pays soit spécialisé ou non. Enfin selon les nouvelles théories du commerce extérieur, la spécialisation intrabranche provoquerait un commerce croisé sans qu'il n'apparaisse d'excédent commercial structurel. On comprend donc l'intérêt d'utiliser un indicateur de spécialisation indépendant du solde commercial. Nous avons choisi l'indice de Michaeli (1962), plus connu sous le nom d'indice d'Aquino (1978).

Plus précisément, on adopte les notations suivantes pour un pays $j$ donné $(1 \leq j \leq P)$ :

- $\left(X_{i j}\right)_{1 \leq i \leq N}$ représente les exportations du pays $j$ dans chacune des $N$ branches retenues ;

- $X_{. j}=\sum_{1 \leq i \leq N} X_{i j}$, le total des exportations du paysj;

- $X_{i .}=\sum_{1 \leq j \leq P}^{1 \leq i \leq N} X_{i j}$, les exportations mondiales dans la branchei ;

- $X_{. .}=\sum_{1 \leq j \leq P} X_{j}$ les exportations mondiales tous produits;

- $\left(M_{i j}\right)_{1 \leq i \leq N}$ représente les importations du paysj dans chacune des $N$ branches ;

- $M_{. j}=\sum_{1 \leq i \leq N} M_{i j}$, le total des importations du paysj;

3 Il existe également des indices de spécialisation de la production calculés à partir de valeurs ajoutées sectorielles. Dans ce qui suit, il s'agit d'un indice de spécialisation interbranche du commerce que l'on nommera indice de spécialisation pour simplifier. 
- $M_{i .}=\sum_{1 \leq j \leq P} M_{i j}$, les importations mondiales dans la branchei ;

- $M_{. .}=\sum_{1 \leq j \leq P} M_{j}$ les importations mondiales tous produits;

- $B_{j}=X_{. j}-M_{. j}$ est la balance commerciale globale du pays $j$. De plus, $X_{i .}=M_{i}$. et $X_{. .}=M_{. .}$

Les variables sont en dollars courants.

L'indice de MICHAELI est alors: $I_{j}=\frac{1}{2} \cdot \sum_{1 \leq i \leq N}\left|\frac{X_{i j}}{X_{. j}}-\frac{M_{i j}}{M_{. j}}\right|$

$I_{j}$ est compris entre 0 et 1 . Plus il se rapproche de 1 et plus les soldes de branche sont déséquilibrés, et donc plus le commerce est spécialisé entre les branches. Une difficulté apparait quant au choix de la désagrégation sectorielle du commerce : un trop petit nombre de branches ne permet pas de faire ressortir de spécialisation sectorielle fine ; un trop grand nombre de branches ne permet pas de distinguer spécialisation interbranche et spécialisation intrabranche.

On peut retrouver cet indice de deux manières. Selon la première, on pose

- $X_{i j}^{\prime}=X_{i j}-\frac{X_{i j}}{X_{. j}+M_{. j}} B_{j}=X_{i j} \cdot \frac{2 \cdot M_{. j}}{X_{. j}+M_{. j}}$ exportations du pays $j$ dans la branche $i$ corrigées d'un termeattribuable au déséquilibre commercial global;

- $M_{i j}^{\prime}=M_{i j}+\frac{M_{i j}}{X_{. j}+M_{. j}} B_{j}=M_{i j} \cdot \frac{2 \cdot X_{. j}}{X_{. j}+M_{. j}}$ importations du pays $j$ dans la branche $i$ corrigées d'un terme attribuable au déséquilibre commercial global;

Les flux ainsi corrigés sont équilibrés: $\sum_{1 \leq i \leq N} X_{i j}^{\prime}=\sum_{1 \leq i \leq N} M_{i j}^{\prime}$

On peut alors montrer que l'indice $I_{j}$ est un indice de Grubel et Lloyd construit à partir des flux corrigés: $I_{j}=\frac{\sum_{1 \leq i \leq N}\left|X_{i j}^{\prime}-M_{i j}^{\prime}\right|}{\sum_{1 \leq i \leq N}\left(X_{i j}^{\prime}+M_{i j}^{\prime}\right)}$. En remontant aux flux initiaux de commerce extérieur l'indice s'écrit encore:

$$
I_{j}=\frac{\sum_{1 \leq i \leq N}\left|X_{i j}-M_{i j}-\frac{X_{i j}+M_{i j}}{X_{. j}+M_{. j}} \cdot B_{j}\right|}{X_{. j}+M_{. j}-\frac{B_{j}^{2}}{X_{. j}+M_{. j}}} \text {. Il est indépendant du solde commercial global }
$$

dans le sens où ce dernier est réparti dans le solde commercial de chaque branche au prorata du poids de celle-ci dans le commerce total.

La deuxième méthode (Aquino) consiste aussi à calculer l'indice de Grubel et Lloyd ci-dessus, mais à partir de flux de commerce corrigés de la façon suivante: les flux sectoriels d'exportations et d'importations sont pondérés, respectivement, par l'inverse du poids des exportations totales et des importations totales dans le commerce total 
$\left(X_{i j}^{\prime}=X_{i j} \cdot\left[\frac{2 \cdot X_{. j}}{X_{. j}+M_{. j}}\right]^{-1}\right.$ et $\left.M_{i j}^{\prime}=M_{i j} \cdot\left[\frac{2 \cdot M_{. j}}{X_{. j}+M_{. j}}\right]^{-1}\right)$. Les flux ainsi corrigés sont également équilibrés.

\subsection{L'indice de dissimilarité du commerce}

A priori, il n'y a aucun désavantage à être fortement spécialisé si la spécialisation résulte d'un avantage comparatif. Cependant, l'écart entre la structure des exportations d'un pays et la structure de la demande internationale constitue aussi un facteur déterminant de la croissance du pays. Pour le voir, il faut raisonner dans une situation d'incertitude où il $\mathrm{y}$ a de nombreux pays et de nombreux produits. Dans ce cadre, la croissance des exportations mondiales et la croissance des exportations d'un paysj sont données par:

$$
\hat{x}=\sum_{1 \leq i \leq N} \frac{X_{i .}}{X_{. .}} \cdot \hat{x}_{i} \text { et } \hat{x}_{j}=\sum_{1 \leq i \leq N} \frac{X_{i j}}{X_{. j}} \cdot \hat{x}_{i j}
$$

où $i$ est l'indice de la branche, $j$ celui du pays et $\hat{x}$ le taux de croissance en volume des exportations.

Alors, pour un niveau fixé de l'incertitude de la demande mondiale, mesurée par la variance ${ }_{x}^{2}$ de la croissance des échanges mondiaux $\hat{x}$, les croissances des exportations mondiales par produit $\hat{x}_{i}$ ne sont pas indépendantes et sont corrélées négativement.

Sous l'hypothèse d'atomicité du pays $j$ ce dernier n'a aucune influence sur ${ }_{x}^{2}$. On suppose qu'il y a un grand nombre de biens et qu'un choc sur les exportations mondiales d'un produit $i$ ne modifie pas les parts de marché des différents pays pour ce produit $\left(\begin{array}{cc}2 \\ \hat{x}_{i j}\end{array}=\begin{array}{l}2 \\ \hat{x}_{i}\end{array}\right)$. On montre alors que l'incertitude sur la croissance des exportations du pays $j$ (mesurée par la variance de $\hat{x}_{j}$ ) est une fonction croissante de ${ }_{x}^{2}$ et de la distance qui sépare la structure de ses exportations de celles de la demande internationale. En conservant les notations du §1.1., une mesure de cette distance est alors donnée par l'indice $A_{j}=\frac{1}{2} \sum_{1 \leq i \leq N}\left|\frac{X_{i j}}{X_{. j}}-\frac{X_{. j}}{X_{. j}}\right|$ que nous appelons indice de dissimilarité du commerce. Il approche son maximum (1) lorsque le pays exporte toujours dans les secteurs où la demande internationale est relativement faible et jamais dans les secteurs où elle est relativement forte. Il atteint son minimum (0) lorsque la structure de ses exportations épouse parfaitement celle de la demande internationale. Si la croissance dépend négativement de l'incertitude sur les exportations, on s'attend à ce qu'elle soit négativement corrélée à l'indice $A_{j}$. 


\subsection{L'indice de croissance de la demande étrangèré}

Dans ce paragraphe, nous voulons étudier les liens entre la croissance d'un pays, la spécialisation de son commerce extérieur et la croissance des échanges internationaux. Pour cela nous définissons l'indice de croissance de la demande étrangère correspondant au pays $j$ par:

$$
C_{j}=\sum_{1 \leq i \leq N}\left(\frac{X_{i j}}{X_{. j}}-\frac{M_{i j}}{M_{. j}}\right) \cdot \hat{z}_{i}
$$

où $\hat{z}_{i}$ est le taux de croissance des exportations mondiales en volume du produit $i$. L'indicateur est nul pour la zone monde. On serait tenté de dire que, lorsqu'il est positif, il est favorable au pays car la croissance des échanges est adaptée à ses exportations. Cependant il n'en est pas toujours ainsi.

Définissons la variation des termes de l'échange du paył par :

$$
P_{j}=\sum_{i}\left(\frac{X_{i j}}{X_{. j}}-\frac{M_{i j}}{M_{. j}}\right) \cdot \hat{p}_{i} \text { où } \hat{p}_{i} \text { est le taux de croissance des prix mondiaux du biens }
$$

et le taux de croissance des exportations nettes par.

$Q_{j}=\sum_{i}\left(\frac{X_{i j}}{X_{. j}}-\frac{M_{i j}}{M_{. j}}\right) \cdot \hat{x}_{i j}$ où $\hat{x}_{i j}$ est le taux de croissance commun des exportations et des importations en volume du pays $j$ dans le bien $i$ (on suppose que les échanges intrabranches restent dans un rapport constant).

La justification de ces indices provient du fait que la variation du taux de couverture $T=\frac{X_{. j}}{M_{. j}}$ s'écrit :

$$
\frac{d T}{T}=P_{j}+Q_{j}
$$

On fait alors les hypothèses suivantes:

- la production est une fonction croissante des termes de l'échange et d'un terme exogène $\hat{y}_{0}$ dépendant des dotations factorielles;

- les demandes d'importations et d'exportations sont telles que les conditions de Marshall-Lerner sont vérifiées : une hausse de la demande extérieure nette provoque une hausse des termes de l'échange;

- l'équilibre est obtenu à taux de couverture constant : $P_{j}+Q_{j}=0$ (une amélioration des termes de l'échange réduit la balance commerciale en volume).

\footnotetext{
${ }^{4}$ Par commodité nous avons conservé la terminologie usuelle. En fait, il s'agit plutôt d'un indice de croissance des échanges internationaux adapté au pays qui se décompose en deux termes : un terme correspondant à la demande étrangère adressée au pays et un terme correspondant à ses exportations nettes.
} 
Dans ce cas il existe en général une corrélation positive entre la croissance de la production $\hat{y}$ et la croissance des échanges internationaux mesurée par $C_{j}$.

Plus précisément, il faut distinguer les chocs de demande et d'offre.

a) Si la croissance des échanges internationaux subit un choc autonome de demande, deux cas peuvent être considérés:

- le choc de croissance de la demande est orienté vers les exportations : l'indice $C_{j}$ s'accroît ex ante. La balance commerciale s'améliore ex ante, les termes de l'échange s'améliorent $\left(P_{j}>0\right)$ ainsi que la production $(\hat{y}>0)$. En revanche les exportations nettes diminuent en volume $\left(Q_{j}<0\right)$ car le pays dépense les suppléments de revenus issus de variations des termes de l'échange. Toutefois si le pays est de petite taille par rapport au marché mondial, cet effet est du second ordre et ne renverse pas le signe de la croissance initiale des échanges internationaux $\left(C_{j}>0\right)$;

- le choc de croissance de la demande est orienté vers les importations : l'indice $C_{j}$ diminue ex ante $\left(C_{j}<0\right)$. La balance commerciale se détériore ex ante et, en vertu des conditions de Marshall-Lerner, les termes de l'échange se détériorent $\left(P_{j}<0\right)$. La situation est donc inverse de la précédente pour les mêmes raisons $\quad \hat{y}<0$ et $\hat{C}_{j}<0$;

b) Si la croissance des échanges internationaux provient d'une croissance autonome du pays, c'est à dire d'un choc d'offre $\left(\hat{y}_{0}>0\right)$, deux cas peuvent encore se présenter:

- la croissance est orientée à l'exportation: dans ce cas, elle augmente l'offre mondiale des biens exportés et réduit les termes de échanges $\left(P_{j}<0\right)$. Les exportations nettes en volume augmentent car le pays réduit ses dépenses à la suite des pertes de revenus liées aux termes de l'échange $\left(Q_{j}>0\right)$ et, de même, les exportations mondiales s'accroissent $\left(C_{j}>0\right)$. Il y a donc à nouveau une corrélation positive entre $\hat{y}$ et $C_{j}$. Toutefois il existe un cas peu probable où la corrélation serait négative $\left(\hat{y}_{0}>0, \hat{\mathrm{y}}<0\right.$ et $\left.C_{j}>0\right)$. Il s'agit de la configuration où la production est très sensible aux termes de l'échange et où leur baisse renverserait l'effet initia $\hat{y}_{0}$.

- la croissance est orientée à l'importation : dans ce cas elle réduit la demande de biens importés du pays et donc augmente les termes de l'échange $\left(P_{j}>0\right)$. Les exportations nettes en volume diminuent car le pays dépense les gains de termes de l'échange $\left(Q_{j}<0\right)$ et la croissance des exportations mondiales diminue $\left(C_{j}<0\right)$. Il y a donc une corrélation négative entre $\hat{y}$ et $C_{j}$. 
La relation entre la croissance et l'indicateur $C_{j}$. est testée dans le $\$ 3.2 .3$. ..

\section{Evolution historique des indices}

Pour calculer les indices, nous avons travaillé dans la nomenclature en 69 produits de la base CHELEM et sur la période 1967-1992. Les indices ne couvrent donc que les produits agricoles et industriels (à l'exclusion de l'or et des pierres précieuses). Ils ne comprennent pas les services et ne permettent pas de discuter de la spécialisation dans ces secteurs. Toutefois la part des services dans les échanges mondiaux reste faible dans la mesure où ces activités sont fortement délocalisées et donnent lieu à des transferts de revenus plutôt qu'à des paiements.

Les résultats sont fournis dans le tableau 1 et dans les graphiques de l'annexe 3. Ils montrent que les pays industriels sont faiblement spécialisés au sens de la spécialisation interbranche, en particulier la France, le Royaume-Uni et l'UEBL. Le Japon fait exception.

Tableau 1 - Les moyennes sur 1967-1992 des trois indices

\begin{tabular}{|c|c|c|c|}
\hline Pays & $\begin{array}{c}\text { Indice de } \\
\text { spécialisation } \\
\text { interbranche }\end{array}$ & $\begin{array}{c}\text { Indice } \\
\text { de dissimilarité du } \\
\text { commerce }\end{array}$ & $\begin{array}{c}\text { Indice de } \\
\text { croissance de la } \\
\text { demande étrangère }\end{array}$ \\
\hline USA & 0,44 & 0,31 & $0,69 \%$ \\
\hline Canada & 0,43 & 0,41 & $-0,50 \%$ \\
\hline France & 0,27 & 0,23 & $0,57 \%$ \\
\hline UEBL & 0,31 & 0,32 & $0,33 \%$ \\
\hline Allemagne & 0,39 & 0,32 & $0,69 \%$ \\
\hline Italie & 0,46 & 0,33 & $1,06 \%$ \\
\hline Pays-Bas & 0,30 & 0,33 & $0,32 \%$ \\
\hline Royaume-Uni & 0,32 & 0,26 & $0,09 \%$ \\
\hline Japon & 0,76 & 0,46 & $2,79 \%$ \\
\hline $\begin{array}{l}\text { Amérique du Sud } \\
\text { (hors Mexique, Brésil) }\end{array}$ & 0,63 & 0,57 & $-0,98 \%$ \\
\hline Venezuela & 0,93 & 0,79 & $-3,50 \%$ \\
\hline $\begin{array}{l}\text { Afrique Noire (hors } \\
\text { Nigeria, Gabon) }\end{array}$ & 0,75 & 0,68 & $-1,90 \%$ \\
\hline Dragons (1) & 0,46 & 0,49 & $0,88 \%$ \\
\hline Tigres (2) & 0,66 & 0,55 & $-1,27 \%$ \\
\hline Mexique & 0,55 & 0,44 & $-2,27 \%$ \\
\hline Brésil & 0,71 & 0,53 & $0,04 \%$ \\
\hline Chine & 0,55 & 0,47 & $-0,75 \%$ \\
\hline Pays de l'Est (3) & 0,35 & 0,35 & $0,20 \%$ \\
\hline URSS & 0,66 & 0,49 & $-1,33 \%$ \\
\hline
\end{tabular}

(1) Corée du Sud, Hongkong, Singapour, Taiwan.

(2) Malaisie, Philippines, Thaïlande.

(3) Hors commerce intra-zone. 


\section{Croissance et spécialisation}

L'Amérique du Sud (hors Mexique et Brésil), le Brésil et l'Afrique Noire sont très spécialisés comme l'ancienne URS\$ et les NPI de deuxième génération (Tigres).

Le Mexique, les Dragons, la Chine sont dans une position intermédiaire.

On retrouve à peu près la même hiérarchie pour l'adéquation à la demande mondiale. Celle-ci est forte pour les pays développés, faible pour l'Afrique Noire, l'Amérique du Sud hormis le Mexique, les NPI de deuxième génération (Tigres). Elle est moyenne pour les anciens NPI (Dragons), le Mexique, la Chine.

La croissance de la demande étrangère adressée aux pays conserve sensiblement la hiérarchie précédente : les pays développés, en particulier le Japon, font face à une demande étrangère globalement croissante sur la période. Cependant le Canada voit cette demande décroître et le Royaume-Uni la voit quasiment stagner. L'augmentation de l'indépendance énergétique des pays développés au cours de la période a fait chuter l'indice des pays producteurs de pétrole dont la plupart, y compris le Mexique, ont eu une production insuffisamment diversifiée pour s'adapter. Les Dragons rejoignent le groupe des pays développés, et la Chine celui des pays en développement.

Les profils d'évolution des deux premiers indices (voir graphiques en annexe 3) permettent d'opérer une partition du monde assez comparable. Les pays industrialisés sont caractérisés par une tendance à la baisse des indices interbranches. Cette tendance a été interrompue entre les années 1974 et 1985 qui correspondent aux chocs pétroliers. Mécaniquement la hausse du prix du pétrole correspond à une modification des termes de l'échange à laquelle les pays industrialisés ont répondu en augmentant le volume de leurs exportations de biens industriels pour équilibrer leur balance commerciale.

Les pays en développement (Amérique du Sud, Afrique Noire) et les Nouveaux Pays Industrialisés (Dragons) ont connu la même tendance à la baisse mais sans qu'on observe de hausse temporaire de l'indice au moment des chocs pétroliers.

$\mathrm{Au}$ contraire, la spécialisation interbranche de l'ex-URSS, des pays de l'Europe de l'Est et de la Chine est restée a peu près constante.

Trois pays ont connu une baisse accélérée de la spécialisation les faisant changer de catégorie. Le Mexique, après une hausse de l'indice de 1977 à 1982 qu'on peut associer aux exportations de pétrole, s'est développé avec une baisse accélérée de sa spécialisation qui l'a fait passer au niveau des pays industrialisés en 1990, ce qui traduit à la fois le contre-choc pétrolier et une nouvelle politique commerciale. L'émergence du Brésil et de la deuxième génération des Nouveaux Pays Industrialisés (Tigres) s'est effectuée de même par une baisse accélérée de la spécialisation qui est passée entre 1967 et 1992 du niveau des pays en développement au niveau intermédiaire correspondant aux Dragons et au Mexique de 1967.

${ }^{5}$ Il est plus difficile de juger les autres pays de l'Est parce qu'on ne dispose pas du commerce interne au COMECON. Or celui-ci organisait les échanges sur la base de la complémentarité des pays. La prise en compte de ce commerce devrait augmenter la spécialisation interbranche de ces pays. 
Les profils des indices de dissimilarité du commerce sont beaucoup plus spécifiques aux pays et aux zones. Les pays développés montrent une détérioration temporaire correspondant aux deux chocs pétroliers (1973-1985). En revanche les Nouveaux Pays Industrialisés, le Brésil, le Mexique connaissent une très forte amélioration de la structure de leurs exportations à la demande mondiale. Cependant l'indice présente un profil très heurté pour le dernier pays avec une détérioration de 1977 à 1982 correspondant au développement des exportations de pétrole et une amélioration très rapide ensuite.

Les pays en développement (Amérique du Sud hors Mexique et Brésil, Afrique noire, Chine) ne montrent aucune tendance de longue période à l'adaptation de leurs exportations à la demande mondiale. Le profil peut cependant être très heurté. Si l'indice est pratiquement constant pour l'Amérique du Sud, pour l'Afrique l'amélioration constatée de 1977 à 1981 et la détérioration de 1982 à 1986 correspondent aux mouvements du prix et de la demande de matières premières. En Chine l'amélioration de 1973 à 1982 et la détérioration de 1982 à 1986 reflètent plutôt la croissance interne.

Les profils d'évolution de l'indice de la croissance de la demande étrangère, contrairement aux autres, ne présentent pas de tendance à la baisse ou à la hausse. En effet pour qu'une telle tendance apparaisse, il faudrait que la croissance de la demande (en volume) adressée au pays s'accélère ou décélère. Le critère discriminant est plutôt la volatilité de l'indice. En effet, les pays en développement, dont le commerce présente une forte spécialisation interbranche, ont des évolutions plus heurtées de leur indice. Un choc de demande ou d'offre, au niveau mondial, est effectivement plus à même de déstabiliser des échanges interbranches que des échanges intrabranches plus équilibrés par nature. Ainsi, les pays de l'OPEP -Algérie, Nigeria, Gabon, Indonésie, Equateur et Venezuela- montrent les évolutions les plus saccadées de tous les pays de l'échantillon. Il en va de même, à un degré légèrement moindre, pour les pays d'Amérique latine dont le commerce est essentiellement tourné vers les exportations de quelques matières premières. Enfin, la volatilité des indices des Dragons et des Tigres est intermédiaire entre celle des pays précédents et celle des pays développés. Ils se distinguent cependant des autres par la tendance à la baisse de la volatilité de leur indice, qui est à rapprocher de leur déspécialisation interbranche.

Les pays développés montrent, dans l'ensemble, des profils relativement plats. L'indice du Japon fait toutefois figure d'exception car, s'il est en moyenne le plus éloigné de celui des pays en développement, son évolution reste semblable à celle de ces derniers. A partir de 1988 sa courbe semble cependant s'aplatir.

Enfin, les anciens pays à économie planifiée sont plus difficilement classables. La Pologne et l'ex-URSS font face à une demande étrangère relativement très instable contrairement à l'ex-Yougoslavie, la Tchécoslovaquie et, mis à part la date du premier choc pétrolier, la Hongrie. La Roumanie, l'ex-RDA et la Bulgarie sont dans une situation intermédiaire.

Ainsi, l'examen des trois indices montre que les pays les plus spécialisés ont aussi les exportations les moins adaptées à la demande mondiale (mais les indices de spécialisation et 


\section{Croissance et spécialisation}

de dissimilarité sont un peu redondants) et font face à une demande étrangère relativement volatile. Ce sont ces mêmes pays qui ont connu la croissance par habitant la plus faible. La croissance des vingt cinq dernières années s'est accompagnée d'une réduction de la spécialisation interbranche et d'une adaptation des exportations à la demande mondiale 6 . Cela peut s'interpréter comme une uniformisation par les échanges mais aussi comme un développement tiré par les exportations et soutenu plus par les rendements d'échelle que par les avantages comparatifs. Enfin, le Japon se distingue très nettement des autres pays développés aussi bien en termes de spécialisation interbranche que de croissance moyenne de la demande étrangère. Il semble toutefois "rentrer dans le rang" en fin de période. Dans le paragraphe suivant nous essayons de répondre aux questions suscitées ici, par une analyse économétrique.

\section{Les résultats économétriques}

Afin d'évaluer l'impact du commerce extérieur sur la croissance nous partons des modèles de croissance à la Solow et nous ajoutons les variables de taux de change, d'ouverture et de spécialisation.

Usuellement, on considère plusieurs facteurs susceptibles d'expliquer la croissance:

- la richesse initiale : selon la théorie de la convergence, dans les régimes de croissance à la Solow, un pays connaît une croissance plus forte lorsqu'il part d'un niveau de production par tête inférieur, parce que la productivité marginale du capital y est plus forte. Nous avons approximé cette variable par le PIB (au prix de la PPA) par habitant en début de période (1967): $\left(\frac{Y_{j t_{0}}^{P A}}{N_{j t_{0}}}\right)$.

- le taux d'investissement : cette variable mesure l'accumulation de capital physique réalisé par le pays. C'est une des variables explicatives du niveau de production par tête qui serait atteint si l'économie continuait à investir indéfiniment à ce taux d'épargne. Elle est mesurée par le rapport investissement/PIB moyen sur la période aux prix internationaux de 1985 (variable $K_{j}$ ).

- le capital humain par tête : cette variable est mesurée par le ratio moyen sur la période du nombre de personnes ayant obtenu un diplôme secondaire rapporté à l'effectif de la population totale (variable $H_{j}$ ). L'introduction de cette variable, qui approxime le capital humain ou le travail qualifié, a deux interprétations différentes qu'il n'est pas possible de discriminer ici:

a) selon le modèle de Römer (1990), le taux de croissance du progrès technique dépend du taux de croissance de la technologie qui s'identifie au nombre de biens utilisables

6 à l'exception de l'Afrique du Sud, du Pakistan, de plusieurs pays producteurs de pétrole (Norvège, Algérie, Nigeria, Gabon) et de la plupart des économies en transition (ex-URSS, Tchécoslovaquie, Hongrie, Pologne, Roumanie, Chine).

${ }^{7}$ l'indice $i$ fait référence au pays. 
comme facteurs intermédiaires de la production de biens finaux. Le taux de croissance du nombre de biens (obtenu par exemple par la recherche-développement) dépend du niveau du travail qualifié.

b) selon les modèles de convergence à la Solow (Barro et Salaa.I.Martin (1991)), le niveau de la productivité globale des facteurs (capital et travail) dépend du niveau du capital humain mesuré ici par le degré moyen d'éducation secondaire de la population. Cette variable intervient donc en niveau dans la dynamique de la convergence.

Nous ne testons donc pas ici un modèle de type Mankiw, Romer et Weil (1991) où la fonction de production est à trois facteurs (capital, travail, capital humain) et où on accumule du capital humain selon un taux d'épargne donné. Dans ce cas, c'est le taux d'investissement du capital humain (variation du capital humain/PIB) qui serait la bonne variable explicative ${ }^{8}$. Cette variable pourrait être approchée par le nombre de personnes scolarisées dans le secondaire rapporté au PIB ou pour éviter un problème de valorisation du capital humain par le taux de scolarisation secondaire (nombre de scolarisés dans le secondaire/nombre de personnes ayant obtenu un diplôme secondaire).

Numériquement et avec les indicateurs retenus, le niveau du capital humain par tête discrimine plus les pays que le taux de croissance du capital humain. Il faut voir là selon nous une donnée factuelle : le capital humain s'accumule très lentement et beaucoup plus lentement que le capital physique. L'évolution du progrès technique dans un pays, dans la période qui nous intéresse, dépend plus de sa capacité à mettre en oeuvre le capital humain existant qu'à l'accumuler.

A ces variables, nous avons ajouté les facteurs dépendant de la politique monétaire des pays. Le premier concerne la sous-évaluation monétaire. Un pays dont le taux de change réel serait inférieur à la PPA corrigée de l'effet Balassa (1964) bénéficierait d'une compétitivité-prix à l'exportation qui devrait favoriser la croissance. Toutefois cet effet n'est réel que si le pays a une certaine maîtrise des prix à l'exportation et peut gagner des parts de marché en écoulant les quantités produites à un prix inférieur à celui de ses concurrents. L'effet dépend donc du degré de spécialisation et de la qualité de cette dernière (matières premières ou biens industriels de grande diffusion). Il est mesuré par le résidu de la régression du taux de change réel moyen sur la période sur le PIB (PPA) moyen par habitant (variable $R_{j}^{\text {Balassa }}$ ). La variable explicative est une approximation du niveau de développement et donc de l'importance de l'effet Balassa.

Le deuxième effet concerne la volatilité des termes de l'échange. Celle-ci devrait constituer un handicap pour le commerce en raison de l'incertitude qu'elle induit sur les rendements pour deux raisons:

\footnotetext{
${ }^{8}$ Le taux de croissance de la productivité du capital et du travail dépend alors du taux de croissance du capital humain.
} 


\section{Croissance et spécialisation}

- la variabilité des prix conduit les exportateurs à arbitrer entre les quantités offertes et le risque associé à ces ventes. Elle les amène à fixer leur offre d'exportation en fonction croissante de la profitabilité espérée et décroissante de la variance des prix à l'exportation.

- la variabilité des prix à l'importation entache d'incertitude les coûts des équipements et matières premières importés, renforçant l'incertitude des rendements et donc limitant l'offre de producteurs ayant de l'aversion pour le risque.

Cet effet est mesuré par le coefficient de variation (écart-type/moyenne) des termes de l'échange (variable $V_{j}$ ).

Nous avons également introduit une variable financière qui mesure les flux de capitaux étrangers entrés dans le pays rapportés au PIB en valeur (en moyenne sur la période). Ces flux correspondent aux prêts, crédits, investissements directs, investissements de portefeuille et transferts sans contrepartie reçus, et sont nets des remboursements de prêts et de désinvestissements. Par l'intermédiaire de cette variable on entend évaluer l'effet sur la croissance du financement externe de l'économie ou des contraintes financières pesant sur les pays en développement. Elle est notée $F_{j}$.

Enfin nous avons introduit les variables spécifiques au commerce international. Celles-ci sont exemptes d'effet conjoncturel en ce sens qu'elles ne dépendent pas de la balance commerciale car nous voulons mesurer l'influence du commerce sur la croissance mais pas de la contrainte extérieure ou des politiques d'excédent durable.

La première question est de savoir si l'engagement dans les échanges mondiaux procure un supplément de croissance. Les arguments sont connus : le commerce mondial procure des avantages comparatifs (commerce interbranche) et permet des économies d'échelle (commerce intrabranche). En revanche, le détournement des productions vers l'exportation peut se faire en prélevant des ressources sur les secteurs de production de biens qui représentent la technique, diminuant ainsi la productivité globale des facteurs. Cependant, cet effet peut disparaître si les exportations autorisent des importations de biens représentant cette technologie. Les conséquences de l'ouverture sur la croissance dépendront donc du poids relatif de ces quatre effets. L'ouverture est mesurée par le ratio : (exportations+importations)/PIB en valeur moyenne sur la périodê (noté $O U V_{j}$ ).

La deuxième question concerne l'influence de la spécialisation. A priori les conséquences en sont incertaines. Les premiers types d'arguments proviennent des modèles dérivés de celui de Ethier (1982) et Romer (1987) selon lesquels la diversification des biens capitaux intermédiaires produits à partir des facteurs primaires procure des rendements croissants dans le secteur des biens finaux et donc une externalité positive à l'économie. Le commerce interbranche concerne les biens finaux entre eux ou les biens finaux contre les biens capitaux pris comme un tout. La spécialisation intrabranche concerne les échanges

${ }^{9}$ Ce ratio est en fait biaisé par la taille du pays, les petits pays étant généralement plus ouverts que les grands (Naudet 1994). Pour en tenir compte, on peut lui substituer le résidu de la régression de $O U V_{i}$ sur l'effectif de la population. Les résultats de la partie III sont alors très peu modifiés. 
entre les biens capitaux ${ }^{10}$. Il en résulte que les échanges intrabranches permettent d'augmenter le nombre de biens capitaux intermédiaires disponibles dans les pays et donc accroissent la productivité du capital. C'est un moyen de diffuser la technologie. En revanche, la spécialisation interbranche, si elle développe les secteurs de biens finaux où le pays a un avantage comparatif, retire des ressources de facteurs non reproductibles des secteurs qui fabriquent les biens capitaux et qui font l'objet de commerce intrabranche. Elle réduit donc la croissance. Ce serait l'inverse si l'avantage comparatif et le commerce interbranche provoquaient le développement du secteur des biens capitaux. Cependant le développement simultané des échanges interbranche et intrabranche aurait un effet inverse sur la croissance mondiale : si la demande mondiale se déplace vers les biens finaux d'un pays qui a un avantage comparatif dans les biens capitaux (pays développés), cela provoque un déplacement de ses ressources vers les biens finaux et donc une baisse du nombre total de biens capitaux dans le monde et une diminution de la croissance mondiale. C'est l'inverse si la demande mondiale se déplace vers les biens finaux d'un pays qui a un avantage comparatif dans ces derniers (pays en voie de développement).

D'autres arguments sont liés à l'apprentissage par la pratique (learning by doing). L'augmentation de la productivité des facteurs dépend du niveau d'activité dans le pays où qu'elle ait lieu. La spécialisation et sa nature serait donc neutre vis-à-vis de la croissance puisqu'elle permettrait de bénéficier des gains d'apprentissage des autres pays par les échanges. Cependant si les gains d'apprentissage sont plus faibles dans les biens finaux, la spécialisation interbranche dans ces secteurs, liée par exemple à un avantage comparatif, se traduit, au cours du temps, par une baisse de la capacité du pays à importer des biens d'équipement, donc une réduction de la croissance du nombre et de la quantité de biens capitaux disponibles, et in fine de la croissance globale. La spécialisation ne doit pas entraver la capacité à importer la technologie. Dans les régressions la spécialisation interbranche est mesurée par l'indicateur du $\$ 1.1$..

La troisième question porte sur les canaux qu'empruntent les échanges commerciaux pour agir sur la croissance. Le commerce international a-t-il joué à travers des effets de demande, ou bien s'est-il développé par des chocs d'offre autonomes? Et parmi ces derniers, peut-on distinguer les pays qui ont fondés leur croissance sur des stratégies de substitution à l'importation, des pays qui ont orienté leur croissance vers l'exportation afin de desserrer leur contrainte financière et de pouvoir importer du capital non produit nationalement. L'indice de croissance de la demande étrangère du $§ 1.3$. est utilisé dans le but de répondre à ces questions.

Enfin, on démontre formellement au §1.2. que l'incertitude sur la croissance des exportations d'un pays est fonction de la dissimilarité entre la structure de ces exportations et celle de la demande internationale. Cette incertitude a-t-elle une influence sur la croissance? L'introduction de l'indice de dissimilarité du commerce vise à répondre à cette question.

\footnotetext{
${ }^{10}$ Les échanges intrabranches entre biens finaux augmentent la diversité des biens de consommation et donc l'utilité des consommateurs ayant du goût pour la diversité . Cependant, ils n'ont aucune influence sur les rendements d'échelle.
} 
Les régressions portent sur 57 pays $^{11}$ et sur la période 1967-1991 ${ }^{12}$. Les sources statistiques sont présentées en annexe I et la variable endogène est le taux de croissance annuel moyen du PIB en volume par habitant du pays : $\left(\frac{Y^{P P A}}{N}\right)$. Les trois indicateurs du $§ I$ sont notés $I_{j}$.

\subsection{L'effet Balassa}

On attribue parfois la compétitivité des pays du Sud sur les marchés du Nord à des politiques de sous-évaluation délibérée du taux de change nominal par rapport à la PPA. L'effet Balassa vient cependant "légitimer" une certaine part de cette sous évaluation en avançant l'argument suivant. Au cours de son processus de développement, un pays réalise des gains de productivité dans son secteur exposé (essentiellement, l'industrie manufacturière) grâce à l'accumulation de capital physique et de capital humain. En revanche, le secteur abrité (principalement, les services) ou traditionnel augmente son niveau de productivité global dans une moindre proportion. Il s'en suit, si l'on suppose que les niveaux de salaire sont sensiblement égaux d'un secteur à l'autre à cause de la mobilité intersectorielle du travail, un accroissement relatif des prix du secteur abrité par rapport à ceux du secteur exposé. Enfin, si l'on admet que le taux de change nominal s'ajuste de façon à égaliser le prix des biens échangés sur les marchés internationaux, alors le prix du PIB d'un pays en développement (moyenne des prix sectoriels) converti au taux de change nominal est nécessairement inférieur au prix du PIB d'un pays développé. Ceci revient encore à dire que le taux de change nominal du pays en développement est inférieur au taux de change PPA : il y a donc sous évaluation par rapport à la PPA, mais celle-ci se trouve en partie justifiée par le degré de développement atteint. Dans le même ordre d'idée, deux pays qui ont atteint un stade de développement équivalent (i.e. les écarts relatifs de prix entre les secteurs sont sensiblement identiques d'un pays à l'autre, de même que la composition sectorielle du PIB) doivent commercer avec un taux de change nominal qui assure la PPA.

Afin d'évaluer cet effet sur notre échantillon de pays, nous avons régressé le taux de change réel $R=\frac{P_{P I B}}{P_{P I B}^{U S}} 13$ sur le PIB PPA par habitant $\left(\frac{Y^{P P A}}{N}\right)$, ratio pris comme indicateur de niveau de développement. Le résidu de la régression représente donc la part de la sousévaluation monétaire que l'on ne peut expliquer par le niveau de développement. Il constitue un indicateur de "dumping" monétaire susceptible de favoriser une croissance tirée par des exportations rendues artificiellement compétitives. Des spécifications arithmétique et logarithmique ont été testées. On a aussi introduit les variables indicatrices des PECO et des pays à monnaies non-convertible. En effet, dans le premier cas on a cherché à isoler les particularités d'une zone dont le commerce avec l'OCDE était très limité et contrôlé. Dans le second cas, un taux de change nominal imposé par les autorités monétaires locales ainsi qu'un contrôle des changes entravent le mouvement d'égalisation des prix des biens

\footnotetext{
${ }^{11} \mathrm{La}$ liste figure en annexe.

12 1967-1990 pour l'ex-RDA.

13 i.e. rapport du prix du PIB du pays sur le prix du PIB d'un pays de référence (USA ici), exprimés dans une monnaie commune.
} 
échangés, si bien que le raisonnement ci-dessus ne peut plus s'appliquer aussi bien. Il s'agit donc de voir si les pays à monnaie non-convertible gênent la régression. Les résultats sont résumés dans le tableau 2.

Les spécifications testées sont:

$R=+\cdot \frac{Y^{P P A}}{N}$ et $\log (R)=+\cdot \log \left(\frac{Y^{P P A}}{N}\right)+\cdot \Uparrow_{z}$.

où $\Uparrow_{z}$ vaut 1 lorsque le paysi appartient à la zone $z$, et 0 sinon.

Tableau 2 - Evaluation de l'effet Balassa ${ }^{14}$

\begin{tabular}{lcccc}
\hline & $1 *$ & $2 * *$ & $3 * *$ & $4 * *$ \\
\hline Constante & 0.41 & -3.54 & -3.53 & -3.63 \\
Pib PPA par habitant & $(11.70)$ & $(-11.88)$ & $(-11.93)$ & $(-12.28)$ \\
& $6.6910^{-5}$ & - & - & - \\
Log(Pib PPA par & $(11.27)$ & & & 0.392 \\
habitant) & - & 0.384 & 0.381 & $(11.07)$ \\
Indicatrice PECO & - & $(10.69)$ & $(10.66)$ & - \\
& - & - & 0.108 & 0.129 \\
Indicatrice pays à & - & - & $(1.35)$ & $(1.85)$ \\
monnaie non- & & & - & 0.683 \\
convertible & 0.692 & 0.669 & 0.674 & 0.21 \\
$\bar{R}^{2}$ & 0.14 & 0.21 & 0.21 & \\
$D$ & & & & \\
\hline
\end{tabular}

NB : les chiffres entre parenthèses représentent les statistiques de Student, $\bar{R}^{2}$ le $R^{2}$ ajusté et $D$ l'écart-type de la régression.

* correspond à la spécification en niveau.

** correspond à la spécification en logarithme.

Les coefficients de l'indicateur de niveau de développement retenu $\left(\frac{Y^{P P A}}{N}\right)$ sont tous très significatifs, et conformément à l'effet Balassa, ils sont positifs. Le résidu est donc d'autant plus petit qu'une monnaie est sous-évaluée par rapport à ce que lui "autoriserait" le niveau de développement du pays où elle circule. Les variables indicatrices améliorent peu la qualité des régressions et ne sont pas significatives au seuil de 5\%. Nous retenons donc pour la suite le résidu de la régression spécifiée en logarithmes sans variable indicatrice.

\footnotetext{
14 A noter que la bonne qualité des régressions est en partie due au fait que, pour 12 des 57 pays de l'étude, les PIB PPA de Chelem sont obtenus à l'aide d'une équation de type Balassa estimée sur les pays déclarants de la base.
} 


\subsection{Etudes économétriques}

On teste ici le modèle de croissance dans lequel chacun des trois indices du §I (notés $I_{j}$ ) intervient successivement comme variable explicative. Deux types de régressions ont alors été effectuées. Les régressions pour lesquelles l'indice $I_{j}$ est décomposé selon les cinq zones Asie, PECO, Afrique, Amérique latine et OCDE, et celles pour lesquelles il a été conservé tel quel. Les deux équations suivantes ont ainsi été estimées à l'aide de la méthode des moindres carrés ordinaires:

$$
\begin{aligned}
\left(\frac{Y^{P P A}}{N}\right)_{j} & =a+b \cdot \log \left(\frac{Y_{j t_{0}}^{P P A}}{N_{j t_{0}}}\right)+c \cdot O U V_{j}+e \cdot R_{j}^{\text {Balassa }}+f \cdot \log \left(H_{j}\right)+g \cdot \log \left(K_{j}\right)+h \cdot \log \left(V_{j}\right)+i \cdot F_{j} \\
& +\left(d_{\text {Asie }} \Uparrow_{\text {Asie }}+d_{P E C O} \Uparrow_{P E C O}+d_{\text {Afrique }} \Uparrow_{\text {Afrique }}+d_{\text {Am.latine }} \Uparrow_{\text {Am.latine }}+d_{O C D E} \Uparrow_{O C D E}\right) \cdot I_{j}
\end{aligned}
$$

et

$$
\left(\frac{Y^{P P A}}{N}\right)_{j}=a^{\prime}+b^{\prime} \cdot \log \left(\frac{Y_{j t_{0}}^{P P_{A}}}{N_{j_{0} t_{0}}}\right)+c^{\prime} \cdot O U V_{j}+d^{\prime} \cdot I_{j}+e^{\prime} \cdot R_{j}^{\text {Balassa }}+f^{\prime} \cdot \log \left(H_{j}\right)+g^{\prime} \cdot \log \left(K_{j}\right)+h^{\prime} \cdot \log \left(V_{j}\right)+i^{\prime} \cdot F_{j}
$$

où $\Uparrow_{z}$ vaut 1 lorsque le pays $j$ appartient à la zone $z$, et 0 sinon.

Afin d'évaluer l'apport de chacun des indices au modèle, nous les avons d'abord exclus des régressions. Le tableau 3 présente les résultats. Ceux-ci font ressortir un noyau stable de variables explicatives, à savoir:

- la richesse initiale;

- l'ouverture de l'économie au commerce extérieur,

- la sous-évaluation du taux de change;

- le capital humain;

- la volatilité des termes del'échange;

A l'exception du résidu Balassa, chaque variable a un coefficient significativement différent de 0 (au seuil de 5\%) et améliore la qualité de la régression, mesurée par le $R^{2}$ ajusté. Le résidu Balassa n'est significatif qu'au seuil de $10 \%$. Le signe du coefficient qui lui est associé (e) est alors négatif, traduisant l'effet favorable d'une sous-évaluation monétaire sur la croissance. Conformément à la théorie de la convergence, la richesse initiale d'un pays contribue d'autant moins à la croissance qu'elle est élevée (coefficient $b$ négatif). En revanche, le taux d'ouverture apparaît comme ayant une contribution positive à l'augmentation du PIB (coefficient $c$ positif). Le détournement d'une partie de la production vers les exportations est donc plus que compensé par les effets favorables de l'ouverture: économies d'échelle, diffusion technologique et augmentation de la diversité des biens capitaux. Toutefois, cet effet globalement positif de l'ouverture peut être réduit par la volatilité des termes de l'échange (coefficient $h$ négatif). En effet, la croissance de production nationale pâtit de l'incertitude sur le prix des biens importés et de l'aversion pour le risque des exportateurs. Enfin, le capital humain participe positivement à la croissance (coefficient $f$ positif). Au total, ces cinq variables réussissent à expliquer $45 \%$ de la croissance des pays de l'étude.

La variable d'entrée de capitaux n'est absolument pas significative et détériore la qualité des régressions. Elle a l'inconvénient de ne pas distinguer entre les flux de capitaux 
qui donnent lieu à des investissements productifs susceptibles d'accélérer la croissance, et les flux plus spéculatifs et instables. Les résultats montrent que les formes de financement ou la contrainte financière ne jouent pas sur la croissance moyenne de la période. Dans notre estimation, cela peut en partie provenir de deux raisons. Tout d'abord notre échantillon contient un grand nombre de pays développés pour lesquels la forme du financement importe peu. En second lieu, les contraintes financières s'exercent en général de manière temporaire sur des périodes de temps qui ne correspondent pas nécessairement à l'intervalle de l'estimation. Enfin, le taux d'investissement en capital physique ne parvient pas à avoir un coefficient significatif. La fiabilité des données d'investissement, en particulier pour les anciens pays à économie planifiée (PECO et Chine), en est sans doute la cause.

En effet, ces pays (à l'exception de l'ex-RDA) se singularisent par des taux de croissance sensiblement inférieurs aux valeurs estimées en raison du faible niveau de richesse initiale et des niveaux relativement élevés de capital humain et d'investissement en capital physique; mais il existe également d'autres causes. Tout d'abord, le coefficient d'échelle de la production peut y être plus faible, ce qui introduit un biais dans la constante du modèle de convergence. Ensuite, dans ces pays, la consommation et la production marchande étaient comprimées, ce qui surévalue mécaniquement le taux d'investissement. Enfin, les monnaies n'étaient pas convertibles, ce qui fait perdre de sa pertinence à l'effet Balassa, d'autant plus que la planification du commerce extérieur visait à obtenir un équilibre des échanges bilatéraux. Pour tenir compte de ces particularités, nous avons introduit la variable indicatrice de la zone PECO+ex-URSS. Elle fait alors grimper le $\bar{R}^{2}$ sensiblement comme le montre le tableau 3, et permet aussi de rendre significatif le coefficient de taux d'investissement, au détriment toutefois du taux d'ouverture.

Tableau 3 - Le modèle de base

\begin{tabular}{|c|c|c|c|c|c|c|c|c|}
\hline & 1 & 2 & 3 & 4 & 5 & 6 & 7 & 8 \\
\hline \multirow[t]{2}{*}{ Constante } & 0.081 & 0.080 & 0.11 & 0.09 & 0.11 & 0.12 & 0.10 & 0.11 \\
\hline & $(4.31)$ & $(4.27)$ & $(5.26)$ & $(4.48)$ & $(4.54)$ & $(5.22)$ & $(4.00)$ & $(4.72)$ \\
\hline \multirow[t]{2}{*}{ Richesse initiale } & -0.009 & -0.008 & -0.013 & -0.013 & -0.013 & -0.014 & -0.013 & -0.011 \\
\hline & $(-3.63)$ & $(-3.53)$ & $(-4.60)$ & $(-4.65)$ & $(-4.51)$ & $(-4.65)$ & $(-4.55)$ & $(-4.23)$ \\
\hline \multirow[t]{2}{*}{ Ouverture } & 0.028 & 0.026 & 0.027 & 0.026 & 0.026 & 0.028 & 0.025 & 0.011 \\
\hline & $(4.56)$ & $(4.11)$ & $(4.48)$ & $(4.44)$ & $(4.02)$ & $(4.57)$ & (3.96) & $(1.60)$ \\
\hline \multirow[t]{2}{*}{ Balassa } & - & -0.011 & -0.017 & -0.016 & -0.017 & -0.017 & -0.016 & -0.015 \\
\hline & & $(-1.14)$ & $(-1.75)$ & $(-1.70)$ & $(-1.78)$ & $(-1.79)$ & $(-1.73)$ & $(-1.76)$ \\
\hline \multirow[t]{2}{*}{ Education second. } & - & - & $9.3410^{-4}$ & $7.4210^{-4}$ & $9.5210^{-4}$ & $9.3810^{-4}$ & $7.6110^{-4}$ & $7.0910^{-4}$ \\
\hline & & & $(2.70)$ & $(2.13)$ & $(2.70)$ & $(2.70)$ & (2.16) & $(2.18)$ \\
\hline \multirow[t]{2}{*}{ Volatilité } & - & - & - & -0.008 & - & - & -0.008 & -0.015 \\
\hline & & & & $(-2.09)$ & & & $(-2.08)$ & $(-3.62)$ \\
\hline \multirow[t]{2}{*}{ Taux d'investissement } & - & - & - & - & 0.004 & - & 0.005 & 0.024 \\
\hline & & & & & $(0.40)$ & & $(0.46)$ & $(2.07)$ \\
\hline \multirow[t]{2}{*}{ Entrées de capitaux } & - & - & - & - & - & -0.054 & - & - \\
\hline & & & & & & $(-0.92)$ & & \\
\hline \multirow{2}{*}{$\begin{array}{l}\text { Indicatrice PECO+ex- } \\
\text { URSS }\end{array}$} & - & - & - & - & - & - & - & -0.022 \\
\hline & & & & & & & & $(-3.18)$ \\
\hline$\overline{\bar{R}}^{2}$ & 0.339 & 0.343 & 0.413 & 0.448 & 0.403 & 0.411 & 0.440 & 0.526 \\
\hline$D$ & 0.015 & 0.015 & 0.014 & 0.013 & 0.014 & 0.014 & 0.014 & 0.012 \\
\hline
\end{tabular}




\section{Croissance et spécialisation}

NB : les chiffres entre parenthèses représentent les statistiques de Student, $\bar{R}^{2}$ le $R^{2}$ ajusté et $D$ l'écart-type de la régression. 


\subsubsection{Régressions avec l'indice de spécialisation interbranche}

Le tableau 4 présente les régressions lorsque l'indice de spécialisation est ajouté au modèle de base. L'indice a un coefficient significativement différent de 0 (au seuil de 5\%) et améliore nettement la qualité des régressions. En l'absence de la variable indicatrice de la zone Est, le $R^{2}$ ajusté augmente de $34 \%$ lorsque l'indice n'est pas désagrégé par zone et de $45 \%$ lorsqu'il l'est. Avec la variable indicatrice, la qualité de la régression augmente de $25 \%$ seulement mais le modèle explique alors $66 \%$ de la croissance. Le coefficient de l'indice de spécialisation, sous sa forme agrégée ou désagrégée, est toujours négatif, traduisant le fait qu'une forte spécialisation interbranche est corrélée avec une faible croissance et inversement. La diversité des biens capitaux que procure le commerce intrabranche serait donc source de croissance conformément au modèle de l'annexe II.

Tableau 4 - Le pouvoir explicatif de l'indice de spécialisation sur la croissance

\begin{tabular}{|c|c|c|c|c|c|c|c|c|c|}
\hline & 1 & 2 & 3 & 4 & 5 & 6 & 7 & 8 & 9 \\
\hline Constante & $\begin{array}{c}0.15 \\
(6.55)\end{array}$ & $\begin{array}{c}0.15 \\
(6.74)\end{array}$ & $\begin{array}{c}0.16 \\
(7.35)\end{array}$ & $\begin{array}{c}0.18 \\
(5.89)\end{array}$ & $\begin{array}{c}0.19 \\
(6.94)\end{array}$ & $\begin{array}{c}0.16 \\
(7.19)\end{array}$ & $\begin{array}{c}0.16 \\
(6.77)\end{array}$ & $\begin{array}{c}0.23 \\
(8.76)\end{array}$ & $\begin{array}{c}0.22 \\
(6.93)\end{array}$ \\
\hline $\begin{array}{l}\text { Richesse } \\
\text { initiale }\end{array}$ & $\begin{array}{l}-0.013 \\
(-5.70)\end{array}$ & $\begin{array}{l}-0.013 \\
(-5.77)\end{array}$ & $\begin{array}{l}-0.016 \\
(-6.27)\end{array}$ & $\begin{array}{l}-0.017 \\
(-6.15)\end{array}$ & $\begin{array}{l}-0.016 \\
(-6.31)\end{array}$ & $\begin{array}{l}-0.017 \\
(-6.10)\end{array}$ & $\begin{array}{l}-0.016 \\
(-5.42)\end{array}$ & $\begin{array}{l}-0.016 \\
(-6.97)\end{array}$ & $\begin{array}{l}-0.016 \\
(-6.29)\end{array}$ \\
\hline Ouverture & $\begin{array}{l}0.027 \\
(5.07)\end{array}$ & $\begin{array}{l}0.024 \\
(4.50)\end{array}$ & $\begin{array}{l}0.025 \\
(4.81)\end{array}$ & $\begin{array}{l}0.025 \\
(4.81)\end{array}$ & $\begin{array}{l}0.022 \\
(3.96)\end{array}$ & $\begin{array}{l}0.026 \\
(4.75)\end{array}$ & $\begin{array}{l}0.020 \\
(3.30)\end{array}$ & $\begin{array}{l}0.009 \\
(1.56)\end{array}$ & $\begin{array}{l}0.008 \\
(1.39)\end{array}$ \\
\hline Balassa & - & $\begin{array}{l}-0.015 \\
(-1.78)\end{array}$ & $\begin{array}{l}-0.019 \\
(-2.25)\end{array}$ & $\begin{array}{l}-0.019 \\
(-2.31)\end{array}$ & $\begin{array}{l}-0.021 \\
(-2.50)\end{array}$ & $\begin{array}{l}-0.019 \\
(-2.25)\end{array}$ & $\begin{array}{l}-0.018 \\
(-1.86)\end{array}$ & $\begin{array}{l}-0.022 \\
(-2.95)\end{array}$ & $\begin{array}{l}-0.021 \\
(-2.80)\end{array}$ \\
\hline Spécialisation & $\begin{array}{l}-0.044 \\
(-4.29)\end{array}$ & $\begin{array}{l}-0.046 \\
(-4.53)\end{array}$ & $\begin{array}{l}-0.042 \\
(-4.20)\end{array}$ & $\begin{array}{l}-0.048 \\
(-3.55)\end{array}$ & $\begin{array}{l}-0.045 \\
(-4.48)\end{array}$ & $\begin{array}{l}-0.041 \\
(-4.05)\end{array}$ & - & $\begin{array}{l}-0.059 \\
(-6.14)\end{array}$ & $\begin{array}{l}-0.055 \\
(-4.41)\end{array}$ \\
\hline $\begin{array}{l}\text { Education } \\
\text { second. }\end{array}$ & - & - & $\begin{array}{c}6.9310^{-4} \\
(2.26)\end{array}$ & $\begin{array}{c}7.3510^{-4} \\
(2.34)\end{array}$ & $\begin{array}{c}7.3110^{-4} \\
(2.40)\end{array}$ & $\begin{array}{c}6.9810^{-4} \\
(2.25)\end{array}$ & $\begin{array}{c}5.6110^{-4} \\
(1.84)\end{array}$ & $\begin{array}{c}7.7510^{-4} \\
(2.88)\end{array}$ & $\begin{array}{c}7.4310^{-4} \\
(2.68)\end{array}$ \\
\hline Volatilité & - & - & - & $\begin{array}{l}0.003 \\
(0.69)\end{array}$ & - & - & - & - & $\begin{array}{c}-2.5310^{-3} \\
(-0.55)\end{array}$ \\
\hline $\begin{array}{l}\text { Taux d'inves- } \\
\text { tissement }\end{array}$ & - & - & - & - & $\begin{array}{l}0.014 \\
(1.48)\end{array}$ & - & - & $\begin{array}{l}0.035 \\
(3.46)\end{array}$ & $\begin{array}{l}0.035 \\
(3.46)\end{array}$ \\
\hline $\begin{array}{l}\text { Entrées de } \\
\text { capitaux }\end{array}$ & - & - & - & - & - & $\begin{array}{l}-0.019 \\
(-0.37)\end{array}$ & - & - & - \\
\hline $\begin{array}{l}\text { Spécialisation } \\
\text { Asie }\end{array}$ & - & - & - & - & - & - & $\begin{array}{l}-0.035 \\
(-2.94)\end{array}$ & - & - \\
\hline $\begin{array}{l}\text { Spécialisation } \\
\text { PECO }\end{array}$ & - & - & - & - & - & - & $\begin{array}{l}-0.053 \\
(-3.32)\end{array}$ & - & - \\
\hline $\begin{array}{l}\text { Spécialisation } \\
\text { Afrique }\end{array}$ & - & - & - & - & - & - & $\begin{array}{l}-0.041 \\
(-3.74)\end{array}$ & - & - \\
\hline $\begin{array}{l}\text { Spécialisation } \\
\text { Am. latine }\end{array}$ & - & - & - & - & - & - & $\begin{array}{l}-0.046 \\
(-4.38)\end{array}$ & - & - \\
\hline $\begin{array}{l}\text { Spécialisation } \\
\text { OCDE }\end{array}$ & - & - & - & - & - & - & $\begin{array}{l}-0.029 \\
(-2.19)\end{array}$ & - & - \\
\hline $\begin{array}{l}\text { Indicatrice } \\
\text { PECO+ex- } \\
\text { URSS }\end{array}$ & - & - & - & - & - & - & - & $\begin{array}{l}-0.021 \\
(-3.88)\end{array}$ & $\begin{array}{l}-0.022 \\
(-3.73)\end{array}$ \\
\hline$\overline{\bar{R}}^{2}$ & 0.500 & 0.520 & 0.555 & 0.550 & 0.565 & 0.547 & 0.598 & 0.661 & 0.656 \\
\hline$D$ & 0.013 & 0.013 & 0.012 & 0.012 & 0.012 & 0.012 & 0.012 & 0.011 & 0.011 \\
\hline
\end{tabular}

NB : les chiffres entre parenthèses représentent les statistiques de Student, $\bar{R}^{2}$ le $R^{2}$ ajusté et $D$ l'écart-type de la régression. 


\section{Croissance et spécialisation}

Toutefois, il convient de nuancer cette conclusion lorsque l'on examine les résidus de l'équation 3. En effet, l'adjonction de indice de spécialisation ne parvient pas à mieux expliquer la diversité des niveaux de croissance que l'on constate dans une zone en développement rapide comme l'Asie. Les écarts entre taux de croissance réel et taux de croissance expliqué ne sont pas réduits dans plusieurs pays : c'est le cas du Japon, où la très forte spécialisation devrait constituer un handicap pour la croissance selon les régressions alors que, dans les faits, elle en serait plutôt le moteur. C'est aussi le cas de l'Inde, de l'Indonésie, de Hongkong, de Singapour et de la Malaisie. Cette constatation est de plus renforcée par le fait que le coefficient de l'indice désagrégé par zone est plus faible pour l'Asie que pour les trois autres zones en développement de l'échantillon (équation 7). En fait, la variable de spécialisation réduit plutôt les résidus des pays développés à l'exception des pays d'Europe du Nord (Irlande, pays scandinaves, Pays-Bas), de l'Australie, de la Nouvelle-Zélande et bien sûr du Japon. Une baisse de notre indicateur signifie, en effet, aussi bien une augmentation du commerce intrabranche de biens d'équipement favorable à la croissance qu'une augmentation du commerce intrabranche de biens de consommation et de matières premières augmentant le bien-être sans affecter la croissance. Pour faire ce distinguo il faudrait restreindre le calcul de l'indice aux biens d'équipement. Dans ce cas le japon, relativement plus importateur de matières premières et de produits agricoles, verrait son indice baisser relativement à celui des autres pays de l'OCDE.

\subsubsection{Régressions avec l'indice de dissimilarité du commerce}

Le tableau 5 présente les régressions lorsque l'indice de dissimilarité du commerce est introduit dans le modèle de base. Le coefficient associé à l'indice est toujours très significatif et négatif. Il montre ainsi que l'incertitude sur les ventes de produits exportés a un impact négatif sur la croissance. Lorsque l'indice est désagrégé, la valeur absolue du coefficient est la plus forte pour les zones Afrique et Amérique latine, grandes pourvoyeuses de matières premières sur la période de l'étude. La grande volatilité des prix et de la demande de ces dernières en est certainement à l'origine. En revanche, le coefficient et l'indice de dissimilarité sont les plus faibles (en valeur absolue) pour les pays d'Asie et de l'OCDE. Les premiers se sont en effet développés (surtout les Dragons) en exportant des produits de consommation courante dont la demande mondiale est forte. Les seconds pèsent d'un poids important dans le commerce international. Dans le second cas, la structure de leur commerce extérieur est proche de celle de la structure mondiale, et dans le premier elle s'en est rapprochée. Il en résulte que la variance de la croissance de leurs exportations est minimum. En outre, la variable de volatilité des termes de l'échange voit la significativité de son coefficient disparaître (comparer tableaux 3 et 5). Cela pourrait signifier qu'on ne peut départager l'effet de l'incertitude monétaire (variable du taux de change) de l'effet de l'incertitude sur la croissance de la demande étrangère. Enfin, la qualité des régressions est très nettement améliorée puisque le $R^{2}$ ajusté s'élève jusqu'à $69 \%$ (équation 8 ). 
Tableau 5 - Le pouvoir explicatif sur la croissance de l'indice de dissimilarité du commerce

\begin{tabular}{|c|c|c|c|c|c|c|c|c|c|}
\hline & 7 & 2 & 3 & 4 & 5 & 6 & 7 & 8 & 9 \\
\hline Constante & $\begin{array}{c}0.15 \\
(7.19)\end{array}$ & $\begin{array}{c}0.15 \\
(7.31)\end{array}$ & $\begin{array}{c}0.17 \\
(8.23)\end{array}$ & $\begin{array}{c}0.19 \\
(6.93)\end{array}$ & $\begin{array}{c}0.19 \\
(7.68)\end{array}$ & $\begin{array}{c}0.18 \\
(8.02)\end{array}$ & $\begin{array}{c}0.17 \\
(7.29)\end{array}$ & $\begin{array}{c}0.22 \\
(9.23)\end{array}$ & $\begin{array}{c}0.21 \\
(7.61)\end{array}$ \\
\hline $\begin{array}{l}\text { Richesse } \\
\text { initiale }\end{array}$ & $\begin{array}{l}-0.014 \\
(-6.22)\end{array}$ & $\begin{array}{l}-0.014 \\
(-6.24)\end{array}$ & $\begin{array}{l}-0.018 \\
(-7.04)\end{array}$ & $\begin{array}{l}-0.018 \\
(-6.94)\end{array}$ & $\begin{array}{l}-0.018 \\
(-7.03)\end{array}$ & $\begin{array}{l}-0.017 \\
(-6.83)\end{array}$ & $\begin{array}{l}-0.016 \\
(-5.70)\end{array}$ & $\begin{array}{l}-0.017 \\
(-7.62)\end{array}$ & $\begin{array}{l}-0.017 \\
(-6.83)\end{array}$ \\
\hline Ouverture & $\begin{array}{l}0.034 \\
(6.52)\end{array}$ & $\begin{array}{l}0.032 \\
(6.02)\end{array}$ & $\begin{array}{l}0.032 \\
(6.46)\end{array}$ & $\begin{array}{l}0.034 \\
(6.44)\end{array}$ & $\begin{array}{l}0.030 \\
(5.74)\end{array}$ & $\begin{array}{l}0.033 \\
(6.37)\end{array}$ & $\begin{array}{l}0.025 \\
(4.23)\end{array}$ & $\begin{array}{l}0.021 \\
(3.90)\end{array}$ & $\begin{array}{l}0.019 \\
(3.15)\end{array}$ \\
\hline Balassa & - & $\begin{array}{l}-0.014 \\
(-1.68)\end{array}$ & $\begin{array}{l}-0.018 \\
(-2.31)\end{array}$ & $\begin{array}{l}-0.019 \\
(-2.37)\end{array}$ & $\begin{array}{l}-0.020 \\
(-2.50)\end{array}$ & $\begin{array}{l}-0.018 \\
(-2.30)\end{array}$ & $\begin{array}{l}-0.017 \\
(-1.79)\end{array}$ & $\begin{array}{l}-0.020 \\
(-2.81)\end{array}$ & $\begin{array}{l}-0.019 \\
(-2.69)\end{array}$ \\
\hline $\begin{array}{l}\text { Indice de } \\
\text { dissimilarité } \\
\text { du commerce. }\end{array}$ & $\begin{array}{l}-0.061 \\
(-4.98)\end{array}$ & $\begin{array}{l}-0.062 \\
(-5.15)\end{array}$ & $\begin{array}{l}-0.059 \\
(-5.16)\end{array}$ & $\begin{array}{l}-0.066 \\
(-4.59)\end{array}$ & $\begin{array}{l}-0.061 \\
(-5.33)\end{array}$ & $\begin{array}{l}-0.058 \\
(-5.00)\end{array}$ & - & $\begin{array}{l}-0.071 \\
(-6.66)\end{array}$ & $\begin{array}{l}-0.065 \\
(-5.01)\end{array}$ \\
\hline $\begin{array}{l}\text { Education } \\
\text { second. }\end{array}$ & - & - & $\begin{array}{c}7.8410^{-4} \\
(2.75)\end{array}$ & $\begin{array}{c}8.4610^{-4} \\
(2.86)\end{array}$ & $\begin{array}{l}8.2510^{-4} \\
(2.89)\end{array}$ & $\begin{array}{l}7.8710^{-4} \\
(2.73)\end{array}$ & $\begin{array}{c}6.7210^{-4} \\
(2.33)\end{array}$ & $\begin{array}{c}8.9810^{-4} \\
(3.49)\end{array}$ & $\begin{array}{c}8.4410^{-4} \\
(3.16)\end{array}$ \\
\hline Volatilité & - & - & - & $\begin{array}{c}3.3610^{-3} \\
(0.82)\end{array}$ & - & - & - & - & $\begin{array}{c}-3.2310^{-3} \\
(-0.77)\end{array}$ \\
\hline $\begin{array}{l}\text { Taux d'inves- } \\
\text { tissement }\end{array}$ & - & - & - & - & $\begin{array}{l}0.012 \\
(1.29)\end{array}$ & - & - & $\begin{array}{l}0.027 \\
(2.97)\end{array}$ & $\begin{array}{l}0.029 \\
(3.05)\end{array}$ \\
\hline $\begin{array}{l}\text { Entrées de } \\
\text { capitaux }\end{array}$ & - & - & - & - & - & $\begin{array}{l}-0.014 \\
(-0.28)\end{array}$ & - & - & - \\
\hline $\begin{array}{l}\text { Dissimilarité } \\
\text { Asie }\end{array}$ & - & - & - & - & - & - & $\begin{array}{l}-0.049 \\
(-3.67)\end{array}$ & - & - \\
\hline $\begin{array}{l}\text { Dissimilarité } \\
\text { PECO }\end{array}$ & - & - & - & - & - & - & $\begin{array}{l}-0.077 \\
(-4.26)\end{array}$ & - & - \\
\hline $\begin{array}{l}\text { Dissimilarité } \\
\text { Afrique }\end{array}$ & - & - & - & - & - & - & $\begin{array}{l}-0.057 \\
(-4.51)\end{array}$ & - & - \\
\hline $\begin{array}{l}\text { Dissimilarité } \\
\text { Am. latine }\end{array}$ & - & - & - & - & - & - & $\begin{array}{l}-0.064 \\
(-5.33)\end{array}$ & - & - \\
\hline $\begin{array}{l}\text { Dissimilarité } \\
\text { OCDE }\end{array}$ & - & - & - & - & - & - & $\begin{array}{l}-0.050 \\
(-3.27)\end{array}$ & - & - \\
\hline $\begin{array}{l}\text { Indicatrice } \\
\text { PECO+ex- } \\
\text { URSS }\end{array}$ & - & - & - & - & - & - & - & $\begin{array}{l}-0.018 \\
(-3.56)\end{array}$ & $\begin{array}{l}-0.020 \\
(-3.48)\end{array}$ \\
\hline $\bar{R}^{2}$ & 0.542 & 0.557 & 0.606 & 0.604 & 0.611 & 0.599 & 0.639 & 0.685 & 0.682 \\
\hline$D$ & 0.012 & 0.012 & 0.011 & 0.011 & 0.011 & 0.011 & 0.011 & 0.010 & 0.010 \\
\hline
\end{tabular}

NB : les chiffres entre parenthèses représentent les statistiques de Student, $\bar{R}^{2}$ le $R^{2}$ ajusté et $D$ l'écart-type de la régression. 


\subsubsection{Régressions avec l'indice de croissance de la demande étrangère}

L'indice est maintenant celui de la croissance de la demande étrangère. Le tableau 6 présente les résultats des régressions. Ceux-ci montrent que, sur l'ensemble des pays de l'échantillon, la croissance est significativement et positivement corrélée à l'indice. Ainsi conformément à l'analyse du §1.3., soit le commerce international joue à travers des effets de demande, soit les politiques de croissance autonomes menées par certains pays sont orientées à l'exportation (on entend par là que le pays accroît sa production dans les secteurs où il est déjà exportateur net). D'autres politiques de croissance autonomes produiraient une corrélation négative. Nous disons qu'elles sont orientées à l'importation en ce sens que le pays se lance dans une production où il est importateur net, comme par exemple une production industrielle visant à se substituer aux importations ou bien à être exportée afin d'assurer des revenus. Ces politiques ont donc été peu ou pas suivies dans notre échantillon.

Lorsque l'indice est désagrégé par zone, il devient non-significatif pour l'Asie, les PECO et l'Afrique mais reste très significatif pour l'Amérique latine et les pays développés. Du point de vue des politiques de croissance menées dans les pays en développement de notre échantillon, il apparaît donc que les pays d'Asie et d'Afrique auraient orienté leur croissance à l'importation alors que l'Amérique latine aurait principalement orienté la sienne à l'exportation. Cela peut parâtre contradictoire a priori. Toutefois, dans les années soixante pour les Dragons, soixante-dix pour les Tigres et quatre-vingt pour les provinces émergentes de Chine (Guangdong, Shenzhen, etc.), l'Asie se lançait dans des stratégies industrielles visant à promouvoir l'exportation de produits manufacturés qui constituaient alors un secteur dans lequel elle était importatrice nette. Comme cela est expliqué ci-dessus, nous qualifions ces politiques de croissance orientée à l'importation : elles produisent une corrélation négative entre la croissance et notre indicateur et amputent donc la significativité du coefficient. A l'inverse, l'Amérique latine, tout en poursuivant des stratégies industrielles de substitution aux importations jusqu'à la crise de la dette de 1982, a surtout misé sur ses exportations de matières premières agricoles ou industrielles, secteur où elle était déjà exportatrice nette. Nous qualifions ces politiques de croissance orientée à l'exportation qui, conformément à l'analyse du §1.3., donnent lieu à une corrélation positive. Le coefficient de la zone Amérique latine conserve donc sa forte significativité.

Enfin, on peut penser que le développement des exportations de produits manufacturés en Tunisie et au Maroc dans les années quatre-vingt (croissance orientée à l'importation selon notre terminologie puisque ces deux pays sont importateurs nets de produits manufacturés) a fait perdre de sa significativité au coefficient de la même façon que pour l'Asie. Cela d'autant plus que ces deux pays représentent le tiers de la zone Afrique de notre échantillon.

Au total, en faisant grimper le $R^{2}$ ajusté à $60 \%$ (équation 5) et même $65 \%$ lorsqu'on introduit l'indicatrice PECO+ex-URSS (équation 10), l'indice exhibe un fort pouvoir explicatif. 
Tableau 6 - Le pouvoir explicatif sur la croissance de l'indice de croissance de la demande étrangère

\begin{tabular}{|c|c|c|c|c|c|c|c|c|c|c|}
\hline & 1 & 2 & 3 & 4 & 5 & 6 & 7 & 8 & 9 & 10 \\
\hline Constante & $\begin{array}{c}0.10 \\
(6.59)\end{array}$ & $\begin{array}{c}0.10 \\
(6.57)\end{array}$ & $\begin{array}{c}0.12 \\
(6.87)\end{array}$ & $\begin{array}{c}0.11 \\
(6.06)\end{array}$ & $\begin{array}{c}0.14 \\
(6.44)\end{array}$ & $\begin{array}{c}0.12 \\
(6.47)\end{array}$ & $\begin{array}{c}0.12 \\
(6.41)\end{array}$ & $\begin{array}{c}0.12 \\
(4.76)\end{array}$ & $\begin{array}{c}0.15 \\
(6.92)\end{array}$ & $\begin{array}{c}0.14 \\
(6.50)\end{array}$ \\
\hline $\begin{array}{l}\text { Richesse } \\
\text { initiale }\end{array}$ & $\begin{array}{l}-0.011 \\
(-5.56)\end{array}$ & $\begin{array}{l}-0.011 \\
(-5.47)\end{array}$ & $\begin{array}{l}-0.014 \\
(-5.66)\end{array}$ & $\begin{array}{l}-0.014 \\
(-5.59)\end{array}$ & $\begin{array}{l}-0.013 \\
(-5.57)\end{array}$ & $\begin{array}{l}-0.014 \\
(-5.43)\end{array}$ & $\begin{array}{l}-0.013 \\
(-5.21)\end{array}$ & $\begin{array}{l}-0.013 \\
(-4.30)\end{array}$ & $\begin{array}{l}-0.013 \\
(-5.39)\end{array}$ & $\begin{array}{l}-0.012 \\
(-5.20)\end{array}$ \\
\hline Ouverture & $\begin{array}{l}0.027 \\
(5.38)\end{array}$ & $\begin{array}{l}0.025 \\
(4.87)\end{array}$ & $\begin{array}{l}0.026 \\
(5.11)\end{array}$ & $\begin{array}{l}0.025 \\
(5.03)\end{array}$ & $\begin{array}{l}0.023 \\
(4.31)\end{array}$ & $\begin{array}{l}0.026 \\
(5.03)\end{array}$ & $\begin{array}{l}0.023 \\
(4.25)\end{array}$ & $\begin{array}{l}0.021 \\
(2.85)\end{array}$ & $\begin{array}{l}0.017 \\
(2.84)\end{array}$ & $\begin{array}{l}0.012 \\
(1.94)\end{array}$ \\
\hline Balassa & - & $\begin{array}{l}-0.011 \\
(-1.36)\end{array}$ & $\begin{array}{l}-0.014 \\
(-1.79)\end{array}$ & $\begin{array}{l}-0.014 \\
(-1.75)\end{array}$ & $\begin{array}{l}-0.016 \\
(-1.98)\end{array}$ & $\begin{array}{l}-0.014 \\
(-1.78)\end{array}$ & $\begin{array}{l}-0.022 \\
(-2.24)\end{array}$ & $\begin{array}{l}-0.017 \\
(-1.78)\end{array}$ & $\begin{array}{l}-0.016 \\
(-2.01)\end{array}$ & $\begin{array}{l}-0.015 \\
(-1.99)\end{array}$ \\
\hline $\begin{array}{l}\text { Indice de } \\
\text { croissance } \\
\text { de la } \\
\text { demande } \\
\text { étr. }\end{array}$ & $\begin{array}{l}0.642 \\
(5.35)\end{array}$ & $\begin{array}{l}0.641 \\
(5.38)\end{array}$ & $\begin{array}{l}0.584 \\
(4.88)\end{array}$ & $\begin{array}{l}0.549 \\
(4.28)\end{array}$ & $\begin{array}{l}0.612 \\
(5.08)\end{array}$ & $\begin{array}{l}0.578 \\
(4.72)\end{array}$ & - & - & $\begin{array}{l}0.627 \\
(5.35)\end{array}$ & $\begin{array}{l}0.524 \\
(4.35)\end{array}$ \\
\hline $\begin{array}{l}\text { Education } \\
\text { second. }\end{array}$ & - & - & $\begin{array}{c}5.8110^{-4} \\
(1.95)\end{array}$ & $\begin{array}{c}5.3510^{-4} \\
(1.76)\end{array}$ & $\begin{array}{c}6.1410^{-4} \\
(2.07)\end{array}$ & $\begin{array}{c}5.8610^{-4} \\
(1.95)\end{array}$ & $\begin{array}{c}6.4210^{-4} \\
(2.02)\end{array}$ & $\begin{array}{c}9.9810^{-4} \\
(2.84)\end{array}$ & $\begin{array}{c}6.6110^{-4} \\
(2.29)\end{array}$ & $\begin{array}{c}5.4410^{-4} \\
(1.94)\end{array}$ \\
\hline Volatilité & - & - & - & $\begin{array}{c}-2.8310^{-5} \\
-0.81)\end{array}$ & - & - & - & - & - & $\begin{array}{c}-9.08 \\
5 \\
(-2.35)\end{array}$ \\
\hline $\begin{array}{l}\text { Taux } \\
\text { d'inves- } \\
\text { tissement }\end{array}$ & - & - & - & - & $\begin{array}{l}0.012 \\
(1.35)\end{array}$ & - & - & $\begin{array}{l}0.012 \\
(0.95)\end{array}$ & $\begin{array}{l}0.021 \\
(2.13)\end{array}$ & $\begin{array}{l}0.027 \\
(2.74)\end{array}$ \\
\hline $\begin{array}{l}\text { Entrées de } \\
\text { capitaux }\end{array}$ & - & - & - & - & - & $\begin{array}{l}-0.015 \\
(-0.30)\end{array}$ & - & - & - & - \\
\hline $\begin{array}{l}\text { Croissance } \\
\text { Asie }\end{array}$ & - & - & - & - & - & - & $\begin{array}{l}0.501 \\
(1.64)\end{array}$ & - & - & - \\
\hline $\begin{array}{l}\text { Croissance } \\
\text { PECO }\end{array}$ & - & - & - & - & - & - & $\begin{array}{l}0.477 \\
(1.15)\end{array}$ & - & - & - \\
\hline $\begin{array}{l}\text { Croissance } \\
\text { Afrique }\end{array}$ & - & - & - & - & - & - & $\begin{array}{l}0.344 \\
(1.53)\end{array}$ & - & - & - \\
\hline $\begin{array}{l}\text { Croissance } \\
\text { Am. latine }\end{array}$ & - & - & - & - & - & - & $\begin{array}{l}0.792 \\
(3.45)\end{array}$ & - & - & - \\
\hline $\begin{array}{l}\text { Croissance } \\
\text { OCDE }\end{array}$ & - & - & - & - & - & - & $\begin{array}{l}0.683 \\
(2.72)\end{array}$ & - & - & - \\
\hline $\begin{array}{l}\text { Indicatrice } \\
\text { PECO+ex- } \\
\text { URSS }\end{array}$ & - & - & - & - & - & - & - & $\begin{array}{l}-0.009 \\
(-1.32)\end{array}$ & $\begin{array}{l}-0.010 \\
(-2.00)\end{array}$ & $\begin{array}{l}-0.018 \\
(-3.04)\end{array}$ \\
\hline $\bar{R}^{2}$ & 0.563 & 0.570 & 0.592 & 0.589 & 0.598 & 0.584 & 0.576 & 0.412 & 0.621 & 0.653 \\
\hline$D$ & 0.012 & 0.012 & 0.012 & 0.012 & 0.011 & 0.012 & 0.012 & 0.014 & 0.011 & 0.011 \\
\hline
\end{tabular}

NB : les chiffres entre parenthèses représentent les statistiques de Student, $\bar{R}^{2}$ le $R^{2}$ ajusté et $D$ l'écart-type de la régression.

\subsection{Comparaison des résultats}

Afin d'évaluer l'apport des indices du commerce extérieur à l'explication de la croissance, nous procédons à une comparaison des contributions des variables d'échange pour les différents modèles (équations 3 des tableaux 3, 4, 5 et 6). La lecture du tableau des contributions ci-dessous donne alors les résultats suivants

- l'ouverture a, relativement à la moyenne mondiale, une contribution positive à la croissance en Asie, en Afrique et dans les pays de l'OCDE. En Amérique latine, dans les pays de l'Est, et en ex-URSS -zones qui sont restées relativement fermées- cette contribution est naturellement faible; 
- la sous-évaluation monétaire influence, dans tous les cas, très peu la croissance. Les sous-évaluations des pays d'Asie et d'Amérique latine de l'échantillon ont légèrement contribué à la croissance de ces zones, alors que les sur-évaluations des monnaies africaines l'ont ralentie. La contribution est quasi-nulle pour les pays développés qui ont gardé -en moyenne sur la période- des taux de change proches de leur valeur de PPA. Enfin, la contribution relative à la zone PECO est plus difficile à qualifier à cause de la particularité de la gestion des taux de change de cette zone;

- la spécialisation interbranche du commerce a un effet important puisqu'il est du même ordre de grandeur que la croissance dans toutes les zones. En Afrique et en Amérique latine la spécialisation a contribué plus que la moyenne au ralentissement de la croissance, alors que dans l'OCDE elle y contribuait moins, l'Asie est dans une position intermédiaire;

- la contribution de la dissimilarité entre le commerce extérieur d'un pays et la demande mondiale s'analyse comme celle de la spécialisation interbranche; elle est toutefois d'amplitude plus élevée;

- enfin, la croissance de la demande étrangère, bien qu'elle soit favorable à la croissance économique (coefficients positifs dans les régressions) y contribue négativement d'une part et peu d'autre part. Cela signifie que la "bonne" spécialisation interbranche -celle qui se produit dans les secteurs à forte croissance- reste l'apanage de quelques pays seulement : essentiellement le Japon, les Dragons et, dans une moindre mesure, quelques pays de l'OCDE. Pour la plupart des pays, la spécialisation interbranche, parce qu'elle est orientée sur des secteurs peu porteurs, est un frein à la croissance économique : c'est surtout le cas pour l'Afrique et l'Amérique latine; c'est moins vrai pour l'Asie dans son ensemble.

En conclusion, il ressort de cette analyse que l'ouverture d'une économie aux échanges internationaux est de nature à favoriser la croissance dans les deux cas suivants

- soit le pays réussit à se positionner sur quelques secteurs où la demande mondiale est forte et parvient à suivre leur évolution ; citons le cas du Japon et des Dragons. Le pays réalise alors une bonne spécialisation interbranche qui tire sa croissance. Il s'en suivra, avec son développement économique, une augmentation naturelle de son commerce intrabranche qui évincera progressivement sa forte spécialisation interbranche initiale

- soit le pays ne parvient pas à se spécialiser dans de tels secteurs et alors il gagnera à développer un commerce fortement intrabranche de biens capitaux, ce qui lui apportera une grande diversité dans ces biens ainsi qu'une forte productivité dans leur production, deux effets très bénéfiques sur sa croissance économique. 
Tableau 7 - Contributions* des variables d'échange dans les différents modèles (équations 3 des tableaux 3, 4, 5 et 6)

\begin{tabular}{|c|c|c|c|c|c|c|c|}
\hline \multirow{2}{*}{\multicolumn{2}{|c|}{ Modèle de base }} & \multicolumn{4}{|c|}{ Zone monde } & \multicolumn{2}{|c|}{ 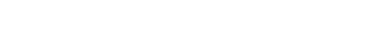 } \\
\hline & & \multicolumn{2}{|c|}{$\begin{array}{l}\text { Avec indice de } \\
\text { spécialisation }\end{array}$} & \multicolumn{2}{|c|}{ Avec indice de dissimilarité } & \multicolumn{2}{|c|}{$\begin{array}{l}\text { Avec indice de croissance de } \\
\text { la demande }\end{array}$} \\
\hline $\begin{array}{l}\text { Variable } \\
\text { endogène }\end{array}$ & $2.42 \%$ & $\begin{array}{l}\text { Variable } \\
\text { endogène }\end{array}$ & $2.42 \%$ & Variable endogène & $2.42 \%$ & Variable endogène & $2.42 \%$ \\
\hline Constante & $10.85 \%$ & Constante & $16.25 \%$ & Constante & $17.44 \%$ & Constante & $11.92 \%$ \\
\hline $\begin{array}{l}\text { Richesse } \\
\text { initiale }\end{array}$ & $10.46 \%$ & $\begin{array}{l}\text { Richesse } \\
\text { initiale }\end{array}$ & $-12.95 \%$ & Richesse initiale & $-13.92 \%$ & Richesse initiale & $-10.74 \%$ \\
\hline Ouverture & $1.09 \%$ & Ouverture & $1.03 \%$ & Ouverture & $1.32 \%$ & Ouverture & $1.04 \%$ \\
\hline Balassa & $0.00 \%$ & Balassa & $0.00 \%$ & Balassa & $0.00 \%$ & Balassa & $0.00 \%$ \\
\hline \multirow[t]{2}{*}{ Educ. second. } & $0.93 \%$ & Educ. second. & $0.69 \%$ & Educ. second. & $0.78 \%$ & Educ. second. & $0.58 \%$ \\
\hline & & Spécialisation & $-2.60 \%$ & $\begin{array}{l}\text { Indice de } \\
\text { dissimilarité }\end{array}$ & $-3.20 \%$ & $\begin{array}{l}\text { Croissance de la } \\
\text { demande }\end{array}$ & $-0.38 \%$ \\
\hline Résidu & $0.00 \%$ & Résidu & $0.00 \%$ & Résidu & $0.00 \%$ & Résidu & $0.00 \%$ \\
\hline
\end{tabular}

\begin{tabular}{|c|c|c|c|c|c|c|c|}
\hline & & & & Zone Asie & & & \\
\hline Modèle de & base & $\begin{array}{l}\text { Avec ind } \\
\text { spécialis }\end{array}$ & $\begin{array}{l}\text { e de } \\
\text { tion }\end{array}$ & Avec indice de di & imilarité & $\begin{array}{r}\text { Avec indice de cr } \\
\text { la deman }\end{array}$ & $\begin{array}{l}\text { ssance de } \\
\text { e }\end{array}$ \\
\hline $\begin{array}{l}\text { Variable } \\
\text { endogène }\end{array}$ & $4.35 \%$ & $\begin{array}{l}\text { Variable } \\
\text { endogène }\end{array}$ & $4.35 \%$ & Variable endogène & $4.35 \%$ & Variable endogène & $4.35 \%$ \\
\hline Constante & $10.85 \%$ & Constante & $16.25 \%$ & Constante & $17.44 \%$ & Constante & $11.92 \%$ \\
\hline $\begin{array}{l}\text { Richesse } \\
\text { initiale }\end{array}$ & $-9.42 \%$ & $\begin{array}{l}\text { Richesse } \\
\text { initiale }\end{array}$ & $-11.66 \%$ & Richesse initiale & $-12.54 \%$ & Richesse initiale & $-9.67 \%$ \\
\hline Ouverture & $1.73 \%$ & Ouverture & $1.62 \%$ & Ouverture & $2.09 \%$ & Ouverture & $1.65 \%$ \\
\hline Balassa & $0.26 \%$ & Balassa & $0.29 \%$ & Balassa & $0.28 \%$ & Balassa & $0.22 \%$ \\
\hline Educ. second. & $0.62 \%$ & Educ. second. & $0.46 \%$ & Educ. second. & $0.52 \%$ & Educ. second. & $0.39 \%$ \\
\hline & & Spécialisation & $-2.85 \%$ & $\begin{array}{l}\text { Indice de } \\
\text { dissimilarité }\end{array}$ & $-3.69 \%$ & $\begin{array}{l}\text { Croissance de la } \\
\text { demande }\end{array}$ & $-0.20 \%$ \\
\hline Résidu & $0.31 \%$ & Résidu & $0.24 \%$ & Résidu & $0.25 \%$ & Résidu & $0.05 \%$ \\
\hline
\end{tabular}

\begin{tabular}{|c|c|c|c|c|c|c|c|}
\hline & & & & Zone PECO & & & \\
\hline Modèle de & base & $\begin{array}{l}\text { Avec ind } \\
\text { spécialis }\end{array}$ & $\begin{array}{l}\text { e de } \\
\text { tion }\end{array}$ & Avec indice de di & imilarité & $\begin{array}{r}\text { Avec indice de cr } \\
\text { la deman }\end{array}$ & $\begin{array}{l}\text { issance de } \\
\text { e }\end{array}$ \\
\hline $\begin{array}{l}\text { Variable } \\
\text { endogène }\end{array}$ & $1.00 \%$ & $\begin{array}{l}\text { Variable } \\
\text { endogène }\end{array}$ & $1.00 \%$ & Variable endogène & $1.00 \%$ & Variable endogène & $1.00 \%$ \\
\hline Constante & $10.85 \%$ & Constante & $16.25 \%$ & Constante & $17.44 \%$ & Constante & $11.92 \%$ \\
\hline $\begin{array}{l}\text { Richesse } \\
\text { initiale }\end{array}$ & $10 . \overline{73} \%$ & $\begin{array}{l}\text { Richesse } \\
\text { initiale }\end{array}$ & $-13.28 \%$ & Richesse initiale & $-14.28 \%$ & Richesse initiale & $-11.02 \%$ \\
\hline Ouverture & $0.30 \%$ & Ouverture & $0.28 \%$ & Ouverture & $0.36 \%$ & Ouverture & $0.29 \%$ \\
\hline Balassa & $-0.15 \%$ & Balassa & $-0.17 \%$ & Balassa & $-0.17 \%$ & Balassa & $-0.13 \%$ \\
\hline Educ. second. & $1.14 \%$ & Educ. second. & $0.85 \%$ & Educ. second. & $0.96 \%$ & Educ. second. & $0.71 \%$ \\
\hline & & Spécialisation & $-2.18 \%$ & $\begin{array}{l}\text { Indice de } \\
\text { dissimilarité }\end{array}$ & $-2.59 \%$ & $\begin{array}{l}\text { Croissance de la } \\
\text { demande }\end{array}$ & $-0.38 \%$ \\
\hline Résidu & $-0.42 \%$ & Résidu & $-0.75 \%$ & Résidu & $-0.73 \%$ & Résidu & $-0.39 \%$ \\
\hline
\end{tabular}




\begin{tabular}{|c|c|c|c|c|c|c|c|}
\hline \multicolumn{8}{|l|}{ 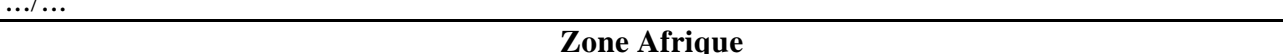 } \\
\hline \multicolumn{2}{|c|}{ Modèle de base } & \multicolumn{2}{|c|}{$\begin{array}{l}\text { Avec indice de } \\
\text { spécialisation }\end{array}$} & \multicolumn{2}{|c|}{ Avec indice de dissimilarité } & \multicolumn{2}{|c|}{$\begin{array}{l}\text { Avec indice de croissance de } \\
\text { la demande }\end{array}$} \\
\hline $\begin{array}{l}\text { Variable } \\
\text { endogène }\end{array}$ & $2.11 \%$ & $\begin{array}{l}\text { Variable } \\
\text { endogène }\end{array}$ & $2.11 \%$ & Variable endogène & $2.11 \%$ & Variable endogène & $2.11 \%$ \\
\hline Constante & $10.85 \%$ & Constante & $16.25 \%$ & Constante & $17.44 \%$ & Constante & $11.92 \%$ \\
\hline $\begin{array}{l}\text { Richesse } \\
\text { initiale }\end{array}$ & $-9.26 \%$ & $\begin{array}{l}\text { Richesse } \\
\text { initiale }\end{array}$ & $-11.46 \%$ & Richesse initiale & $-12.33 \%$ & Richesse initiale & $-9.51 \%$ \\
\hline Ouverture & $1.22 \%$ & Ouverture & $1.15 \%$ & Ouverture & $1.48 \%$ & Ouverture & $1.16 \%$ \\
\hline Balassa & $-0.39 \%$ & Balassa & $-0.44 \%$ & Balassa & $-0.42 \%$ & Balassa & $-0.33 \%$ \\
\hline \multirow{2}{*}{ Educ. second. } & $0.29 \%$ & Educ. second. & $0.22 \%$ & Educ. second. & $0.25 \%$ & Educ. second. & $0.18 \%$ \\
\hline & & Spécialisation & $-3.54 \%$ & $\begin{array}{l}\text { Indice de } \\
\text { dissimilarité }\end{array}$ & $-4.29 \%$ & $\begin{array}{l}\text { Croissance de la } \\
\text { demande }\end{array}$ & $-1.28 \%$ \\
\hline Résidu & $-0.61 \%$ & Résidu & $-0.07 \%$ & Résidu & $-0.03 \%$ & Résidu & $-0.05 \%$ \\
\hline \multirow{2}{*}{\multicolumn{2}{|c|}{ Modèle de base }} & \multicolumn{4}{|c|}{ Zone Amérique latine } & & \\
\hline & & \multicolumn{2}{|c|}{$\begin{array}{l}\text { Avec indice de } \\
\text { spécialisation }\end{array}$} & \multicolumn{2}{|c|}{ Avec indice de dissimilarité } & \multicolumn{2}{|c|}{$\begin{array}{l}\text { Avec indice de croissance de } \\
\text { la demande }\end{array}$} \\
\hline $\begin{array}{l}\text { Variable } \\
\text { endogène }\end{array}$ & $1.14 \%$ & $\begin{array}{l}\text { Variable } \\
\text { endogène }\end{array}$ & $1.14 \%$ & Variable endogène & $1.14 \%$ & Variable endogène & $1.14 \%$ \\
\hline Constante & $10.85 \%$ & Constante & $16.25 \%$ & Const: & $17.44 \%$ & Const & $11.92 \%$ \\
\hline $\begin{array}{l}\text { Richesse } \\
\text { initiale }\end{array}$ & $-\overline{24 \%}$ & $\begin{array}{l}\text { Richesse } \\
\text { initiale }\end{array}$ & $-12.68 \%$ & Richesse initiale & $-13.64 \%$ & Richesse initiale & $-10.52 \%$ \\
\hline Ouverture & $0.72 \%$ & Ouverture & $0.67 \%$ & Ouverture & $0.87 \%$ & Ouverture & $0.68 \%$ \\
\hline Balassa & $0.23 \%$ & Balassa & $0.26 \%$ & Bala & $0.25 \%$ & Balassa & $0.20 \%$ \\
\hline \multirow[t]{2}{*}{ Educ. second. } & $0.58 \%$ & Educ. second. & $0.43 \%$ & Educ. second. & $0.49 \%$ & Educ. second. & $0.36 \%$ \\
\hline & & Spécialisation & $-3.34 \%$ & $\begin{array}{l}\text { Indice de } \\
\text { dissimilarité }\end{array}$ & $-3.90 \%$ & $\begin{array}{l}\text { Croissance de la } \\
\text { demande }\end{array}$ & $-1.14 \%$ \\
\hline Résidu & $-1.01 \%$ & Résidu & $-0.46 \%$ & Résidu & $-0.38 \%$ & Résidu & $-0.37 \%$ \\
\hline \multirow{2}{*}{\multicolumn{2}{|c|}{ Modèle de base }} & \multicolumn{4}{|c|}{$\begin{array}{c}\text { Zone pays développés } \\
\end{array}$} & & \\
\hline & & \multicolumn{2}{|c|}{$\begin{array}{l}\text { Avec indice de } \\
\text { spécialisation }\end{array}$} & \multicolumn{2}{|c|}{ Avec indice de dissimilarité } & \multicolumn{2}{|c|}{$\begin{array}{c}\text { Avec indice de croissance de } \\
\text { la demande }\end{array}$} \\
\hline $\begin{array}{l}\text { Variable } \\
\text { endogène }\end{array}$ & $2.44 \%$ & $\begin{array}{l}\text { Variable } \\
\text { endogène }\end{array}$ & $2.44 \%$ & Variable endogène & $2.44 \%$ & Variable endogène & $2.44 \%$ \\
\hline Constante & $10.85 \%$ & Constante & $16.25 \%$ & Constante & $17.44 \%$ & Constante & $11.92 \%$ \\
\hline $\begin{array}{l}\text { Richesse } \\
\text { initiale }\end{array}$ & $11.29 \%$ & $\begin{array}{l}\text { Richesse } \\
\text { initiale }\end{array}$ & $-13.98 \%$ & Richesse initiale & $-15.03 \%$ & Richesse initiale & $-11.60 \%$ \\
\hline Ouverture & $1.14 \%$ & Ouverture & $1.07 \%$ & Ouverture & $1.38 \%$ & Ouverture & $1.09 \%$ \\
\hline Balassa & $-0.06 \%$ & Balassa & $-0.07 \%$ & Balassa & $-0.07 \%$ & Balassa & $-0.05 \%$ \\
\hline \multirow[t]{2}{*}{ Educ. second. } & $1.30 \%$ & Educ. second. & $0.97 \%$ & Educ. second. & $1.09 \%$ & Educ. second. & $0.81 \%$ \\
\hline & & Spécialisation & $-2.12 \%$ & $\begin{array}{l}\text { Indice de } \\
\text { dissimilarité }\end{array}$ & $-2.64 \%$ & $\begin{array}{l}\text { Croissance de la } \\
\text { demande }\end{array}$ & $0.02 \%$ \\
\hline Résidu & $0.49 \%$ & Résidu & $0.32 \%$ & Résidu & $0.26 \%$ & Résidu & $0.25 \%$ \\
\hline
\end{tabular}

* en moyenne sur la zone considérée

\section{Conclusion}

L'étude économétrique de la liaison entre la croissance et le commerce extérieur montre les résultats suivants:

- Les pays fortement ouverts sont ceux qui ont connu la croissance la plus forte. 
- La croissance est corrélée positivement à celle des échanges internationaux. Cela signifie soit que le commerce international joue à travers des effets de demande, soit que la croissance autonome des pays est orientée à l'exportation. Les stratégies de croissance orientée à l'importation -comme celles de substitution aux importations ou celles de promotion des exportations manufacturières dans des pays importateurs nets de produits industriels- ont joué un rôle minoritaire sur la période considérée. Toutefois, rares sont les pays (essentiellement le Japon, les Dragons et, dans une moindre mesure, quelques pays de l'OCDE) qui ont pu bénéficier de la forte croissance de la demande internationale qu'ont connu certains secteurs : la spécialisation interbranche de la plupart des pays n'a en effet pas été orientée vers de tels secteurs ; la spécialisation interbranche a alors constitué un frein à la croissance.

- La spécialisation interbranche est corrélée négativement avec la croissance et les pays à forte croissance ont connu une réduction au cours du temps de cette forme de spécialisation. D'ailleurs les chocs pétroliers correspondent à une période de moindre croissance associée à une reprise temporaire de la spécialisation interbranche. Les progrès de productivité du travail passent donc en partie par les échanges intrabranches et intraindustriels qui permettent de diversifier les biens intermédiaires incorporant le progrès technique. Le Japon et les Dragons font exception dans la mesure où ils ont réussi à développer une spécialisation interbranche dans les quelques secteurs qui ont connu un développement rapide.

- En revanche les effets de la politique monétaire sont moins nets. Tout d'abord l'incertitude sur les termes de l'échange n'apparaît pas comme une variable significative. Ensuite, la sous-évaluation du taux de change est un facteur explicatif de la croissance, mais à faible contribution, surtout lorsqu'on prend soin de distinguer les anciens pays de l'Est dont la monnaie n'était pas convertible.

\section{L'analyse pourrait être étendue dans plusieurs directions}

- en introduisant la dynamique dans le modèle et en effectuant des régressions sur données de panel;

- en étudiant la causalité entre la croissance et les indices du commerce extérieur afin de savoir si le commerce international, de par sa structure sectorielle, provoque la croissance ou bien s'il en est la conséquence;

- en recalculant l'indice de spécialisation interbranche sur les produits manufacturés uniquement, ou mieux, sur les biens d'équipement. En effet ce sont ces derniers qui sont susceptibles de permettre les gains de productivité source de croissance. L'indice, tel qu'il est calculé ici, mélange commerce intrabranche de biens de consommation et commerce intrabranche de biens capitaux. 


\section{Références bibliographiques}

A. AQUINO (1978) : "Intra-Industry Trade and Inter-Industry Specialization as Concurrent Sources of International Trade in Manufactures", Weltwirtschaftliches Archiv, vol. 114, pp. 275-296.

B. BALASSA (1964) : "The Purchasing Power Parity Doctrine: a Reappraisal", Journal of Political Economy, Décembre, 72, pp. 586-596.

R.J. BARRO (1991): "Economic Growth in a Cross Section of Countries", Quarterly Journal of Economics, n 106, pp. 407-443.

R.J. BARRO et J.W. LEE (1994): "Data Set for a Panel of 138 Countries".

R.J. BARRO et X. SALA.I.MARTIN (1991) : "Convergence across States and Regions", Brooking Paper on Economic Activity, ${ }^{\circ} 1$.

D. DOLLAR (1992) : "Outward-Oriented Developing Economies Really Do grow more rapidely: evidence from 95 LDC's, 1976-1985", The University of Chicago Press.

W. ETHIER (1982) : "National and International Returns to Scale in the Modern Theory of International Trade", American Economic Review, june, vol 72, pp. 389-405.

H.G. GRUBEL et P.J. LLOYD (1975): "Intra-Industry Trade. The Theory and Measurement of International Trade in Differentiated Products", London, England.

G. MANKIW, D. ROMER et D. WEIL (1992): "A Contribution to the Empirics of Economic Growth", Quarterly Journal of Economics, May.

M. MICHAELY (1962): "Concentration in International Trade", Contributions to economic analysis, vol 28, Amsterdam.

D. NAUDET (1994): "Une analyse du degré d'ouverture internationale", Economie Internationale, $\mathrm{N}^{\circ}$ 58-2, pp. 53-81.

P. ROMER (1987) : "Growth based on increasing returns due to specialisation", American Economic Review, May, pp. 56-62.

P. ROMER (1990) : "Endogenous Technological Change", Journal of Political Economy, octobre, pp. 71-102.

S. VONA (1991) : "On the Measurement of Intra-Industry Trade: Some Futher Thoughts", Weltwirschaftliches Archiv, 127, 4, pp. 678-699. 


\section{Annexe 1}

Les séries utilisées dans les régressions de la partie III proviennent des sources statistiques suivantes:

- les PIB PPA, les effectifs de population, les taux de change réels, les flux de capitaux et les flux de commerce extérieur par branche et par pays sont extraits de Chelem

- les indices de prix du commerce extérieur servant à déflater les flux en valeur dans l'indice de §1.3. sont ceux du modèle Mimosa du Cepii. Ils n'existent que pour le secteur manufacturier, le secteur agro-alimentaire, le secteur des matières premières industrielles et celui de l'énergie. On a donc dû affecter à chacune des 69 branches de Chelem l'indice du prix du secteur la contenant;

- les ratios : effectif de la population ayant un diplôme secondaire / effectif total sont issus de la base de données de R. J. Barro et J. Lee (1994) (variable SECCxx)

- les ratios d'investissement en capital physique sont extraits des World Tables (1994) et de "Historically Planned Economies, a Guide to the Data" de la Banque mondiale

- les séries des termes de l'échange sont extraites des IFS (juin 1994) du FMI

Tableau 7 - Pays de la régression et variables indicatrices des zones Asie, PECO, Afrique, Amérique latine et OCDE

\begin{tabular}{|l|c|c|c|c|c|}
\hline \multicolumn{1}{|c|}{ PAYS } & $\Uparrow_{\text {Asie }}$ & $\Uparrow_{\text {PECO }}$ & $\Uparrow_{\text {Afrique }}$ & $\Uparrow_{\text {Am.latine }}$ & $\Uparrow_{O C D E}$ \\
\hline ETATS-UNIS & 0 & 0 & 0 & 0 & 1 \\
CANADA & 0 & 0 & 0 & 0 & 1 \\
FRANCE & 0 & 0 & 0 & 0 & 1 \\
ALL.FEDERALE & 0 & 0 & 0 & 0 & 1 \\
ITALIE & 0 & 0 & 0 & 0 & 1 \\
PAYS-BAS & 0 & 0 & 0 & 0 & 1 \\
JAPON & 0 & 0 & 0 & 0 & 1 \\
AFR. DU SUD & 0 & 0 & 0 & 0 & 1 \\
MEXIQUE & 0 & 0 & 0 & 1 & 0 \\
BRESIL & 0 & 0 & 0 & 1 & 0 \\
INDONESIE & 1 & 0 & 0 & 0 & 0 \\
INDE & 1 & 0 & 0 & 0 & 0 \\
EX-URSS & 0 & 1 & 0 & 0 & 0 \\
CHINE & 1 & 0 & 0 & 0 & 0 \\
ROYAUME-UNI & 0 & 0 & 0 & 0 & 1 \\
\hline
\end{tabular}




\begin{tabular}{|c|c|c|c|c|c|}
\hline 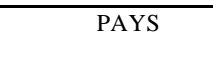 & $\Uparrow_{\text {Asie }}$ & $\Uparrow_{P E C O}$ & $\Uparrow_{\text {Afrique }}$ & $\Uparrow_{\text {Am.latine }}$ & $\Uparrow_{O C D E}$ \\
\hline IRLANDE & 0 & 0 & 0 & 0 & 1 \\
\hline DANEMARK & 0 & 0 & 0 & 0 & 1 \\
\hline FINLANDE & 0 & 0 & 0 & 0 & 1 \\
\hline NORVEGE & 0 & 0 & 0 & 0 & 1 \\
\hline SUEDE & 0 & 0 & 0 & 0 & 1 \\
\hline AUTRICHE & 0 & 0 & 0 & 0 & 1 \\
\hline SUISSE & 0 & 0 & 0 & 0 & 1 \\
\hline ESPAGNE & 0 & 0 & 0 & 0 & 1 \\
\hline GRECE & 0 & 0 & 0 & 0 & 1 \\
\hline PORTUGAL & 0 & 0 & 0 & 0 & 1 \\
\hline TURQUIE & 0 & 0 & 0 & 0 & 1 \\
\hline ISRAEL & 0 & 0 & 0 & 0 & 1 \\
\hline EX-YOUGOSLAV & 0 & 1 & 0 & 0 & 0 \\
\hline AUSTRALIE & 0 & 0 & 0 & 0 & 1 \\
\hline NLLE-ZELANDE & 0 & 0 & 0 & 0 & 1 \\
\hline COREE DU SUD & 1 & 0 & 0 & 0 & 0 \\
\hline HONGKONG & 1 & 0 & 0 & 0 & 0 \\
\hline SINGAPOUR & 1 & 0 & 0 & 0 & 0 \\
\hline TAIWAN & 1 & 0 & 0 & 0 & 0 \\
\hline VENEZUELA & 0 & 0 & 0 & 1 & 0 \\
\hline EQUATEUR & 0 & 0 & 0 & 1 & 0 \\
\hline ALGERIE & 0 & 0 & 1 & 0 & 0 \\
\hline MAROC & 0 & 0 & 1 & 0 & 0 \\
\hline TUNISIE & 0 & 0 & 1 & 0 & 0 \\
\hline EGYPTE & 0 & 0 & 1 & 0 & 0 \\
\hline NIGERIA & 0 & 0 & 1 & 0 & 0 \\
\hline GABON & 0 & 0 & 1 & 0 & 0 \\
\hline MALAISIE & 1 & 0 & 0 & 0 & 0 \\
\hline PHILIPPINES & 1 & 0 & 0 & 0 & 0 \\
\hline THAILANDE & 1 & 0 & 0 & 0 & 0 \\
\hline ARGENTINE & 0 & 0 & 0 & 1 & 0 \\
\hline CHILI & 0 & 0 & 0 & 1 & 0 \\
\hline COLOMBIE & 0 & 0 & 0 & 1 & 0 \\
\hline PEROU & 0 & 0 & 0 & 1 & 0 \\
\hline PAKISTAN & 1 & 0 & 0 & 0 & 0 \\
\hline BRUNEI & 1 & 0 & 0 & 0 & 0 \\
\hline BULGARIE & 0 & 1 & 0 & 0 & 0 \\
\hline TCHECOSLOVAQ & 0 & 1 & 0 & 0 & 0 \\
\hline HONGRIE & 0 & 1 & 0 & 0 & 0 \\
\hline POLOGNE & 0 & 1 & 0 & 0 & 0 \\
\hline ROUMANIE & 0 & 1 & 0 & 0 & 0 \\
\hline RDA & 0 & 1 & 0 & 0 & 0 \\
\hline
\end{tabular}




\section{Annexe 2}

\section{Dérivation de l'équation estimée au paragraphe 3.2.1.}

1) On suppose que le capital est constitué de A biens intermédiaires de quantité $X_{i}, i \in[1, A]$. La fonction de production macro-économique dépend de la diversité des biens intermédiaires. Elle est homogène de degré 1 dans le travail et la liste des inputs de biens capitaux :

$$
Y=N \int_{0}^{A} \frac{\left(X_{i}\right) d i}{N}
$$

où $Y$ est la production, $N$ l'emploi, $X_{i}$ la quantité de bien capital $i$. Si on suppose que les biens capitaux sont homogènes, le capital peut s'agréger sous la forme

$$
K=\int_{0}^{A} X_{i} d i=A X
$$

En supposant que tous les biens capitaux sont en quantité égale (en choisissant des unités adéquates), la fonction de production devient

$$
\text { (1) } \quad Y=A^{1-} K N^{1-}
$$

2) On suppose que la croissance du nombre de biens capitaux est une fonction croissante du capital humain $H$, du degré d'ouverture du pays $O U V$ qui permet de les importer et, à degré d'ouverture fixé, de la part du commerce industriel intrabranche (c'est à dire une fonction décroissante de l'indice de spécialisation interbranche $I_{S P E}$ (défini dans le $\S 1.1$.$) .$

$$
a=\frac{\dot{A}}{A}=f\left(\stackrel{(+)}{H}, \stackrel{(+)}{U} V, \stackrel{(-)}{I}_{S P E}\right)
$$

3) Les conditions d'accumulation sont les suivantes

- l'investissement est donné par la fonction d'épargne $\mathrm{I}=\mathrm{sY}$;

- l'accumulation du capital total est : $\frac{\dot{K}}{K}=\frac{I}{K}-\quad$ où $\quad$ est le taux de dépréciation commun à tous les biens de capitaux;

- la croissance de la population est exogène: $\frac{\dot{N}}{N}=n$.

4) La croissance équilibrée à taux constant est alors donnée par le quadruplet $(\bar{Y}, A, \bar{K}, \bar{N})$ où :

$$
g=\frac{\dot{\bar{Y}}}{\bar{Y}}=(1-\quad) a+g_{K}+(1-\quad) n
$$




\section{Croissance et spécialisation}

mais en outre:

(4) $g_{K}=\frac{\dot{\bar{K}}}{\bar{K}}=s \frac{\bar{Y}}{\bar{K}}-=s\left(\frac{A \bar{N}}{\bar{K}}\right)^{1-}-$

Elle n'existe que si:

(5)

$$
\frac{\dot{\bar{K}}}{\bar{K}}=g_{K}=a+n
$$

d'où :

(6) $g=g_{K}=a+n$

et :

(7) $\left(\frac{\bar{K}}{A N}\right)^{1-}=\frac{s}{a+n+}$

5) En supposant qu'on se trouve toujours au plein emploi $(N=\bar{N})$, la production vaut :

(8) $\quad Y=A^{1-} K \bar{N}^{1-}$

et la croissance du capital:

(9) $\frac{\dot{K}}{K}=s\left(\frac{A \bar{N}}{K}\right)^{1-}-$

L'écart entre la croissance et la croissance équilibrée s'écrit donc en utilisant (4), (5), (6), (7), (8), et (9) :

$$
\begin{aligned}
& \frac{\dot{Y}}{Y}-n-a=\left(\frac{\dot{K}}{K}-a-n\right)=(a+n+)\left[\left(\frac{\bar{K}}{K}\right)^{1-}-1\right] \\
& \frac{\dot{Y}}{Y}-n-a=(a+n+)\left[\left(\frac{\bar{Y}}{Y}\right)^{\frac{1-}{-}}-1\right]
\end{aligned}
$$

En posant $y=\log \frac{Y}{N}$ et $\bar{y}=\log \frac{\bar{Y}}{\bar{N}}$, et en supposant que $\bar{Y} / Y$ est voisin de 1 , on obtient :

$$
\frac{\dot{Y}}{Y}-n=a+(1-\quad)(a+n+)(\bar{y}-y)
$$




$$
\begin{aligned}
& \text { ou encore: } \\
& \frac{\dot{Y}}{Y}-n=a+(a+n+\quad)\left[(1-\quad) \log A+\log \frac{I}{Y}-(1-\quad) y-\log (a+n+\quad)\right]
\end{aligned}
$$

C'est cette équation jointe avec l'équation (2) que nous avons estimée en la linéarisant et en supposant qu'on pouvait l'appliquer sur une période longue. Les taux de croissance, comme le taux d'épargne, sont les valeurs moyennes (sur la période 1967-1991), alors que la production par tête, $y$, et le niveau de progrès technique $A$ sont les valeurs initiales (en 1967). La production initiale par tête $y$ provoque une convergence alors que la croissance du progrès technique $a$ (donc le capital humain et le commerce international) peut induire une divergence. 


\section{Annexe 3}

\section{Graphiques}

1. Indice de spécialisation intrabranche du commerce

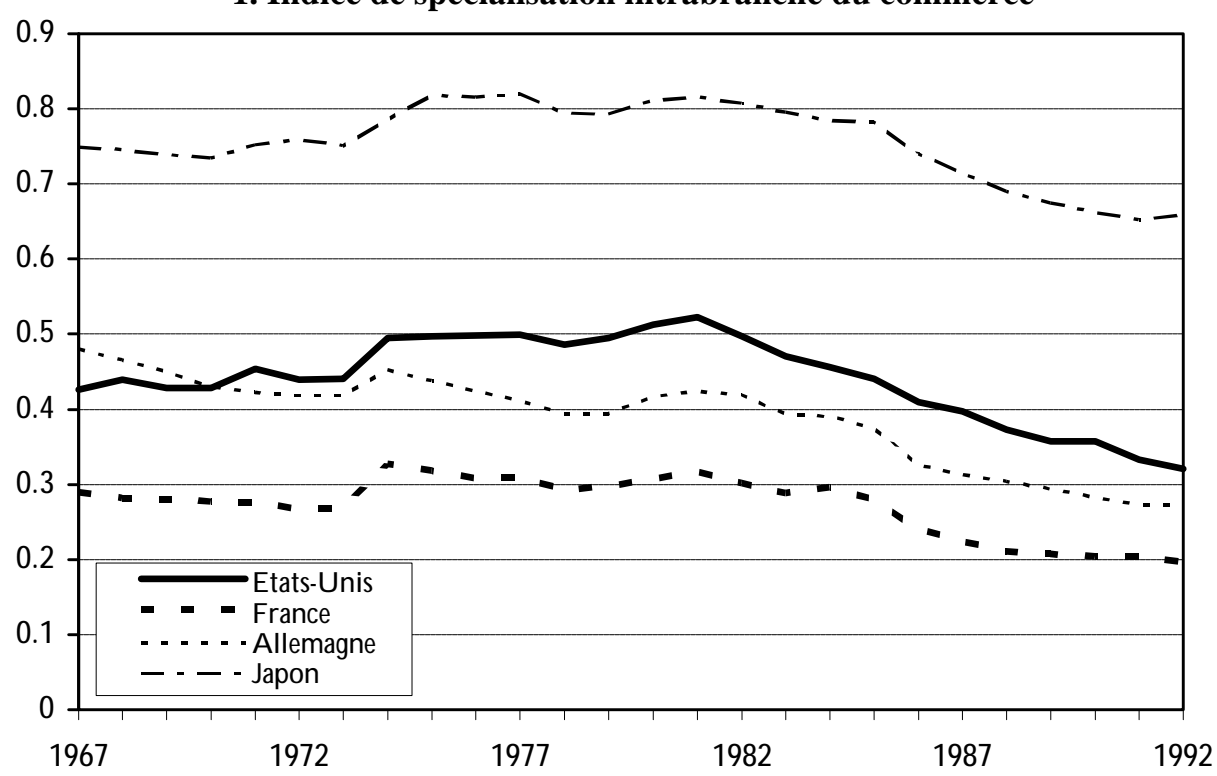

2. Indice de spécialisation intrabranche du commerce

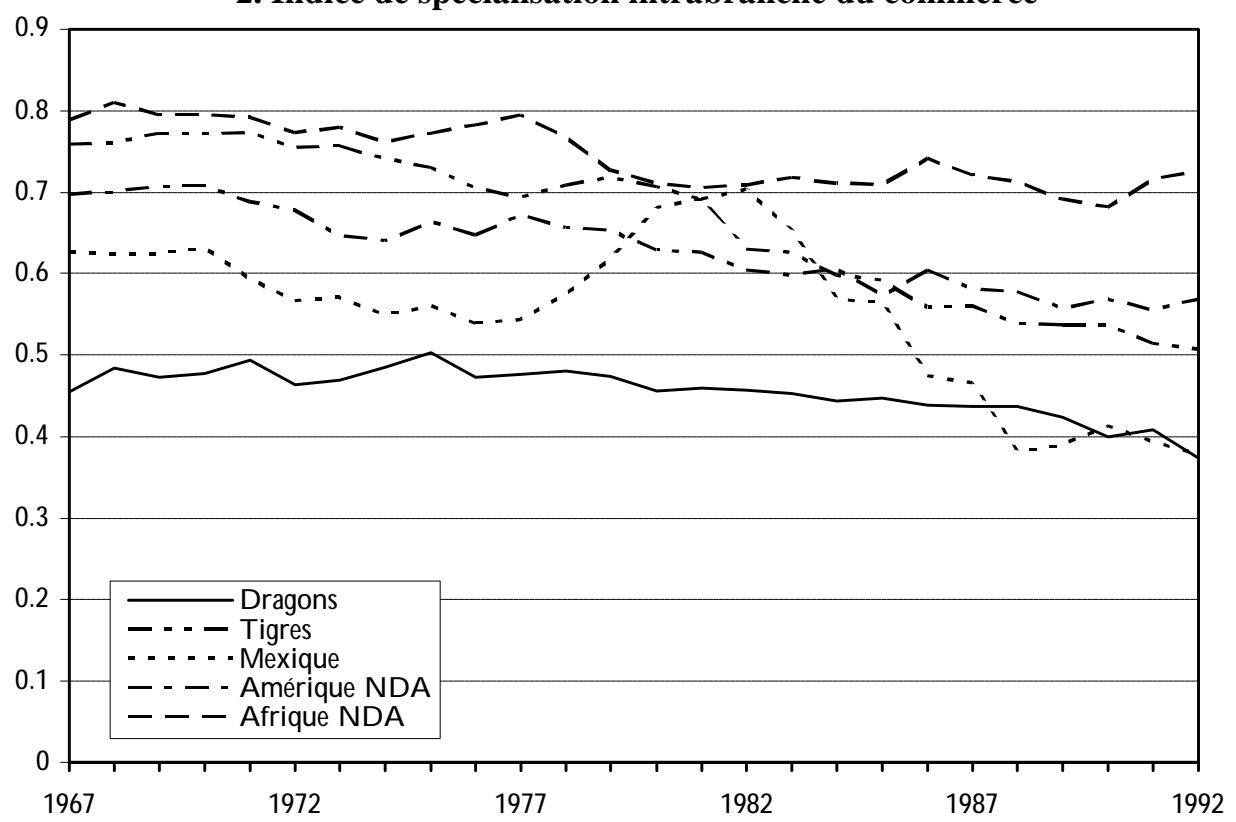

$\mathrm{Nb}$ : Amérique NDA = Amérique latine hors Equateur, Vénézuela, Mexique, Brésil. 
Afrique NDA = Afrique Noire hors Nigéria, Gabon, Afrique du Sud. 


\section{Indice de dissimilarité}

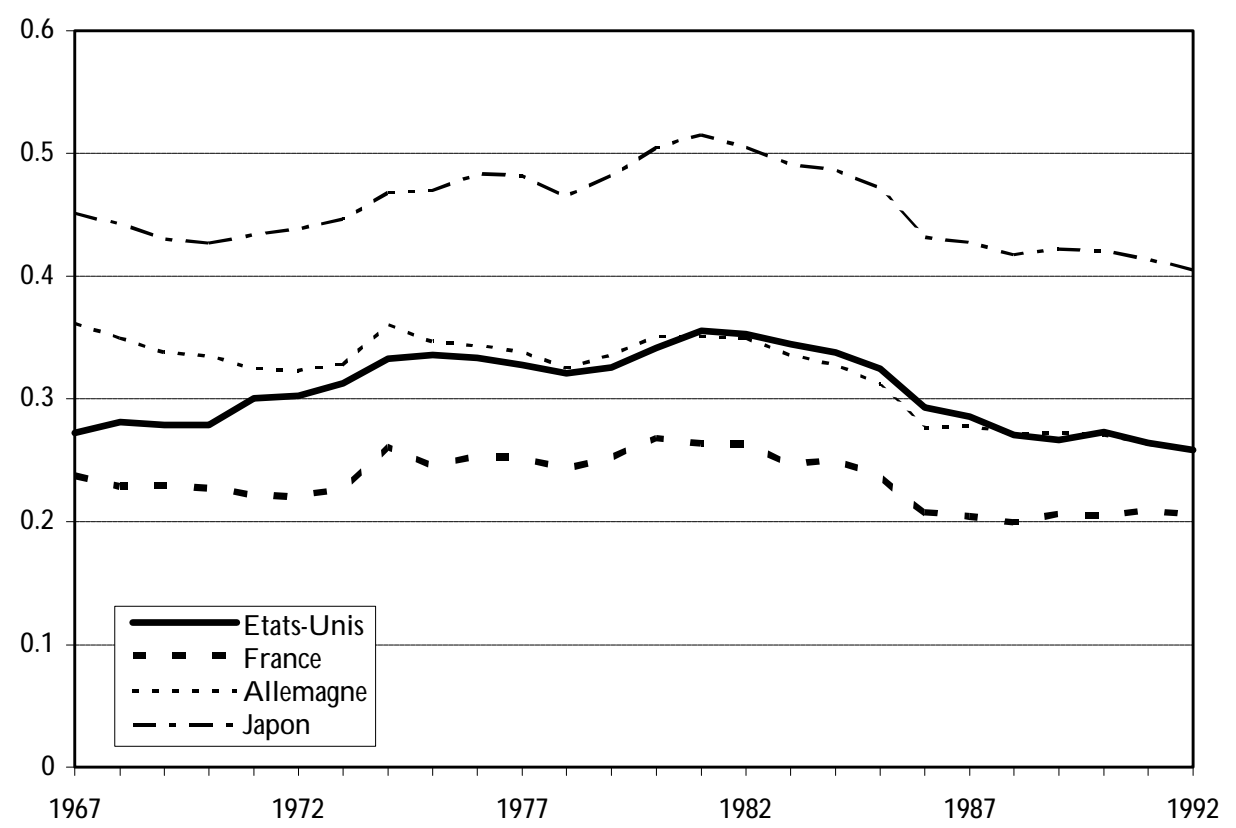

4. Indice de dissimilarité

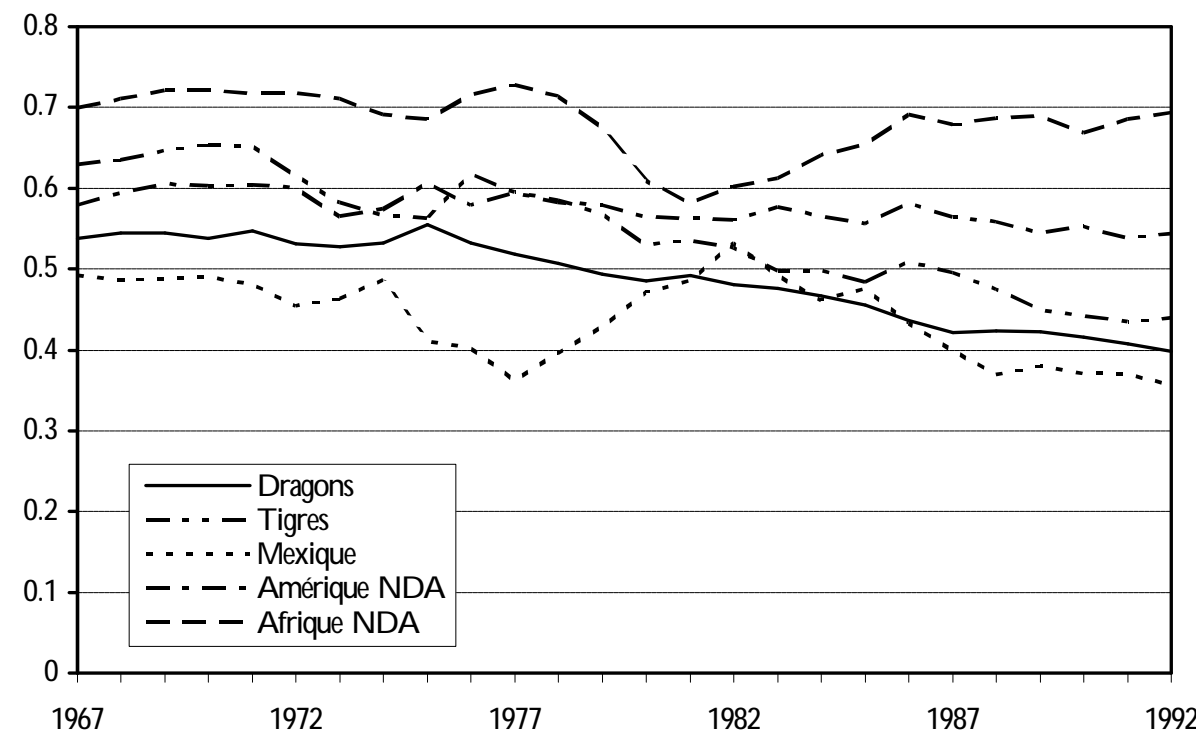

$\mathrm{Nb}$ : Amérique NDA = Amérique latine hors Equateur, Vénézuela, Mexique, Brésil. Afrique NDA = Afrique Noire hors Nigéria, Gabon, Afrique du Sud. 


\section{Indice de croissance de la demande étrangère}

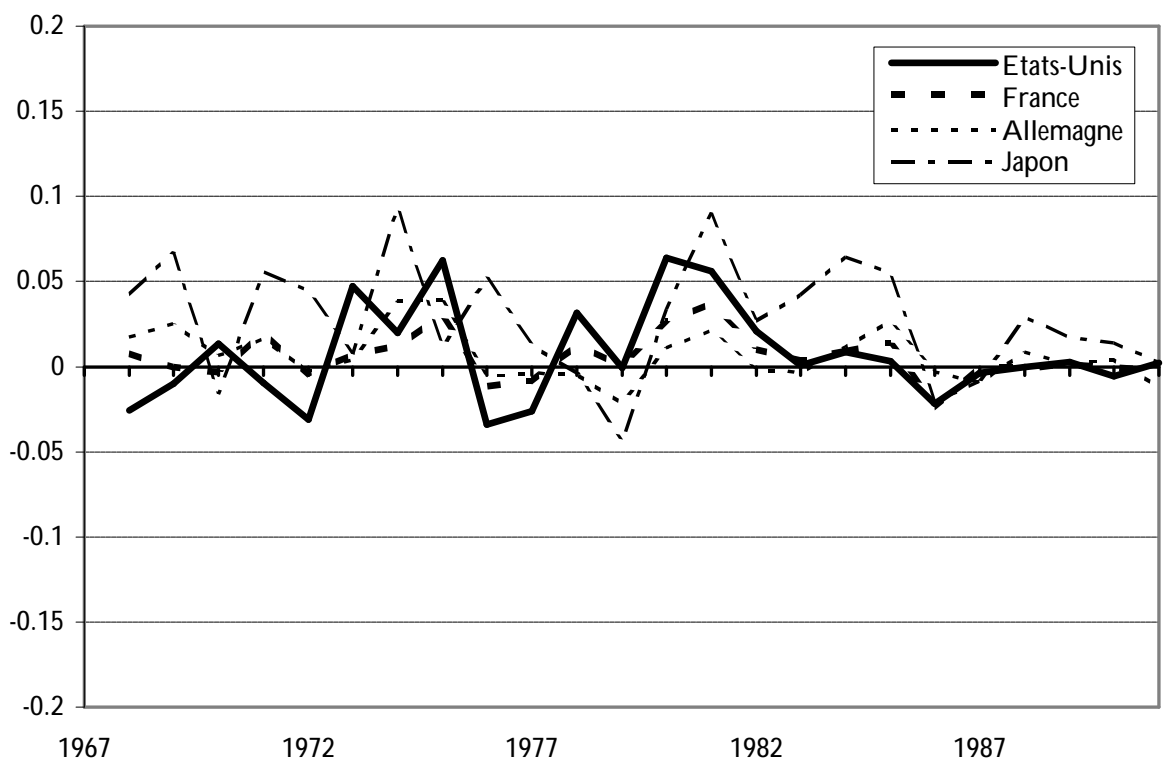

6. Indice de croissance de la demande étrangère

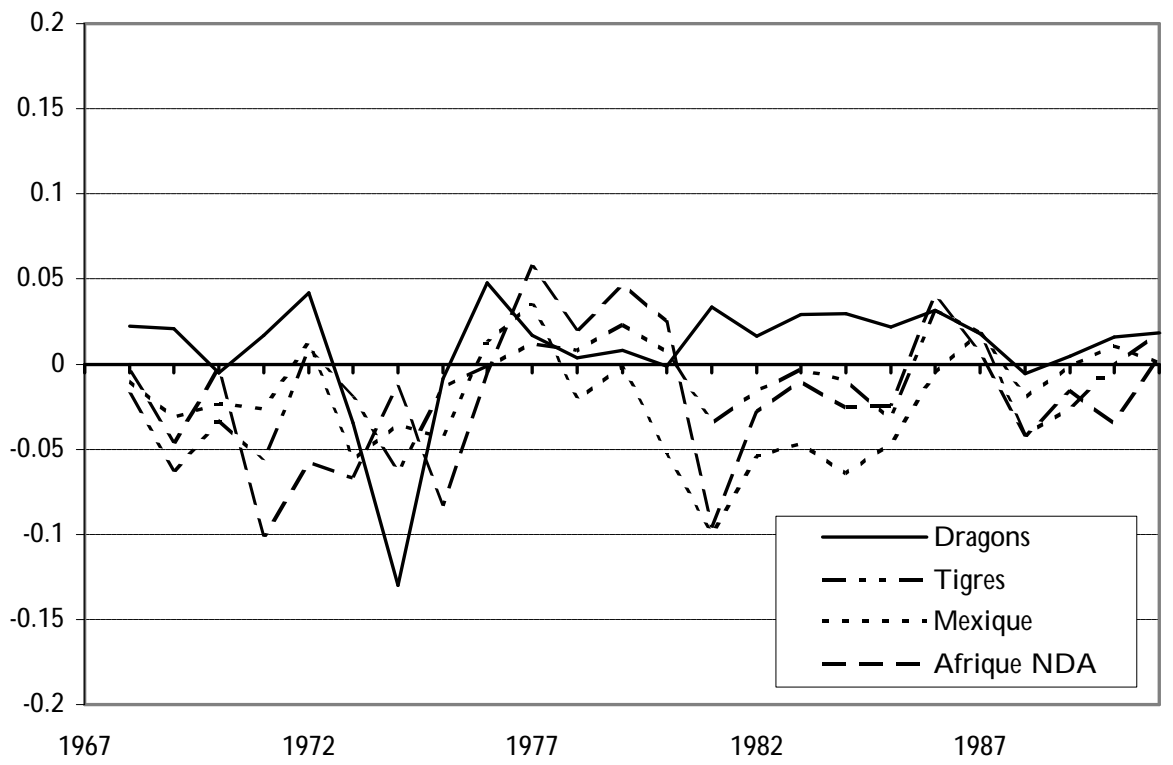

$\mathrm{Nb}$ : Amérique NDA = Amérique latine hors Equateur, Vénézuela, Mexique, Brésil. 


\section{Liste des documents de travail du CEPII}

\section{4}

"The international Monetary System: in search of New Principles", Michel Aglietta, document de travail $n^{\circ} 94-11$, septembre.

"French and German Productivity Levels in Manufacturing: a Comparison Based on the Industry of Origin Method", Deniz Unal-Kesenci et Michael Freudenberg, document de travail $n^{\circ} 94-10$, septembre.

"La réunification allemande du point de vue de la politique économique", Agnès Bénassy et Pierre Villa, document de travail $n^{\circ}$ 94-09, septembre.

"Commerce international, emploi et salaires", Olivier Cortes et Sébastien Jean, document de travail $n^{\circ}$ 94-08, août.

"La fonction de consommation sur longue période en France", Pierre Villa, document de travail $n^{\circ}$ 94-07, juillet.

"Réglementation et prise de risque des intermédiaires financiers : la crise des prix d'actifs au début des années 1990", Benoit Mojon,document de travail $n^{\circ}$ 94-06, juillet.

"Turquie : d'une stabilisation à l'autre", Isabelle Bensidoun, document de travail $n^{\circ}$ 94-05, juillet.

"Economic Policy Strategies to Fight Mass Unemployment in Europe: an Appraisal.", Henri Delessy et Henri Sterdyniak,document de travail $n^{\circ}$ 94-04, juillet.

"Transmission de la politique monétaire et crédit bancaire, une application à cinq pays de l'OCDE", Fernando Barran, Virginie Coudert et Benoît Mojon, document de travail $n^{\circ} 94$ 03 , juin.

"Indépendance de la banque centrale et politique budgétaire", Agnès Bénassy et Jean Pisani-Ferry, document de travail $n^{\circ} 94-02$, juin.

"Les systèmes de paiements dans l'intégration européenne", Michel Aglietta, document de travail $n^{\circ}$ 94-01, mai.

1993

"Crises et cycles financiers : une approche comparative", Michel Aglietta, document de travail $\mathrm{n}^{\circ}$ 93-05, octobre.

$\overline{15}$ Les documents de travail sont diffusés gratuitement sur demande au CEPII au 48426414 ; une liste des documents de travail 1984-1994 est également disponible à ce numéro. 
"Regional and World-Wide Dimensions of Globalization", Michel Fouquin, document de travail $\mathrm{n}^{\circ}$ 93-04, septembre.

"Règle, discrétion et régime de change en Europe", Pierre Villa, document de travail $\mathrm{n}^{\circ}$ 9303, août.

"Crédit et dynamiques économiques", Michel Aglietta, Virginie Coudert, Benoît Mojon, document de travail $\mathrm{n}^{\circ}$ 93-02, mai.

"Les implications extérieures de l'UEM", Agnès Bénassy, Alexander Italianer, Jean PisaniFerry, document de travail ${ }^{\circ}$ 93-01, avril.

1992

"Pouvoir d'achat du franc et restructuration industrielle de la France 1960-1991", Gérard Lafay, document de travail ${ }^{\circ}$ 92-04, décembre.

"Le Franc: de l'instrument de croissance à la recherche de l'ancrage nominal", Michel Aglietta, document de travail n ${ }^{\circ}$ 92-03, décembre.

"Comportement bancaire et risque de système", Michel Aglietta, document de travail n 92 02, mai.

"Dynamiques macroéconomiques des économies du sud: une maquette représentative", Isabelle Bensidoun, Véronique Kessler,document de travail ${ }^{\circ}$ 92-01, mars.

1991

"Europe de l'Est et URSS : niveaux de production et de consommation en Europe de l'Est et comparaisons avec l'Europe de l'Ouest", Françoise Lemoine, document de travail ${ }^{\circ}$ 91-04, décembre.

"Europe de l'Est, URSS, Chine : la montée des déséquilibres macroéconomiques dans les

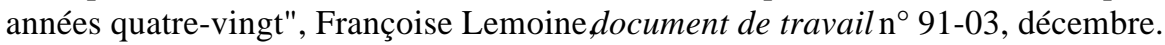

"Ordre monétaire et banques centrales", Michel Aglietta, document de travail n 91-02, mars.

"Epargne, investissement et système financier en Chine", Françoise Lemoine, document de travail $\mathrm{n}^{\circ}$ 91-01, février. 